\title{
Renormalizable SU(5) completions of a Zee-type neutrino mass model
}

\author{
Krešimir Kumerički, Timon Mede, and Ivica Picek \\ Department of Physics, Faculty of Science, University of Zagreb, \\ P.O. Box 331, HR-10002 Zagreb, Croatia
}

(Received 12 January 2018; published 7 March 2018)

\begin{abstract}
We explore the potential of a selected model of radiative neutrino masses to be implemented in a renormalizable SU(5) unification framework. The Zee-type model under consideration uncovers the SU(5) representations in which the new fields are embedded and which may contain also other light states leading to the unification of gauge couplings. We perform an exhaustive search which reveals specific patterns of new states and demonstrate that such patterns are consistent with a general choice of relevant scalar potential. It turns out that all of the specific scenarios which lead to successful unification include the colored scalars testable at the LHC.
\end{abstract}

DOI: 10.1103/PhysRevD.97.055012

\section{INTRODUCTION}

Despite all the phenomenological success of the Standard Model (SM), certain theoretical and experimental issues like neutrino masses, dark matter, charge quantization, hierarchy problem etc. seem to indicate the need to go beyond its well-established framework. The ultraviolet completions motivated by neutrino mass models may address the open questions and pave the road beyond the Standard Model (BSM). For example, the neutrino masses in canonical type-I [1-5], type-II [6-11], and typeIII [12] tree-level seesaw models percolate down from a single scale that may be linked to the unification point of SM gauge couplings, hinted first within the SU(5) grand unified theory (GUT) of Georgi and Glashow [13]. After realizing that there is no single gauge coupling crossing in this simplest GUT, it was noticed that augmenting the SM by the second Higgs doublet and the corresponding supersymmetric (SUSY) partners enables a successful minimal SUSY SM (MSSM) unification [14]. A decisive role [15] played by incomplete (or split) irreducible representations (irreps) $\mathbf{5}_{H}$ in the MSSM unification success, motivated the corresponding non-SUSY attempts to cure the crossing problem [16,17] with just six copies of the SM Higgs doublet field and nothing more. Still, the scale of such unifications would be too low.

Further studies of unification in the context of nonSUSY SU(5) GUTs employed incomplete SU(5) irreps which contain new states introduced by tree-level seesaw

Published by the American Physical Society under the terms of the Creative Commons Attribution 4.0 International license. Further distribution of this work must maintain attribution to the author(s) and the published article's title, journal citation, and DOI. Funded by SCOAP . models. The studies in [18-20] employed adjoint SU(5) representation $\mathbf{2 4}_{F}$ which contains both the fermion singlet and the $\mathrm{TeV}$-scale fermion triplet fields providing a low scale hybrid of type-I and type-III seesaw models. Similarly, Refs. [21-23] employed $\mathbf{1 5}_{S} \mathrm{SU}(5)$ representation with the $\mathrm{TeV}$-scale complex scalar triplet, employed in the type-II seesaw mechanism.

When considering possible GUT embeddings of a radiative neutrino mass generating mechanism, we opt for genuine radiative Zee-type models, genuine in the sense that no additional symmetries are required to make them the dominant contribution to neutrino mass. At the same time, by avoiding fermion singlets we are choosing the SU(5) embedding and discard the $\mathrm{SO}(10)$ one. The first one-loop model proposed by Zee [24] has introduced only new scalar fields, the charged singlet and the second complex doublet, which do not lead to competing treelevel seesaw mechanisms. The embedding of the original Zee model in the renormalizable non-SUSY SU(5) setup has been studied in [25].

Our focus here will be on the variant of the Zee model presented in [26], which in the following we call the BPR model. It keeps the Zee's charged scalar singlet, but a real scalar triplet replaces Zee's second Higgs doublet. Finally, the BPR model introduces three copies of vectorlike lepton doublet fields which, if embedded in split $\mathbf{5}_{F}$, may influence the gauge running as twelve Higgs doublets. Let us note that besides the genuine one-loop model [26] there exist three-loop radiative neutrino models [27,28], where an automatic protection from the tree-level or lower loop contributions has been achieved by introducing appropriate larger weak multiplets. However, the appearance of the $\sim 10^{6} \mathrm{GeV}$ Landau pole (LP) for the $\mathrm{SU}(2)_{L}$ gauge coupling $g_{2}$ [29] eliminates these models from a unification framework. In contrast, as demonstrated in [30], 
the BPR one-loop model with the scalar triplet as the largest weak representation exhibits, in addition to the absence of LP, perturbativity and stability up to the Planck scale. Therefore, we proceed here with the study of the gauge coupling unification in the context of the BPR loop model [26] for which the above requirements with respect to Yukawa and quartic couplings may remain valid when including extra color octet or color sextet scalar fields [31]. As it will turn out, adding these fields may be crucial to achieve the proper gauge unification.

In Sec. II we first present the set of BSM particles from the neutrino model [26], dubbed BPR particles, and then present the gauge-unification conditions which the newly introduced states have to satisfy. In Sec. III, we will study the conditions under which the gauge couplings unify, and the particle spectra which make a proper unification possible. Then in Sec. IV we will show that the appropriate particle spectra are consistent with the scalar potential of our SU(5) GUT scenarios. We conclude in Sec. V. The details of the algorithm for search are given in Appendix A and the details of SU(5) representations in Appendix B.

\section{BPR MODEL FROM GUT PERSPECTIVE}

\section{A. BPR-model states}

We adopt a simple and predictive $\mathrm{TeV}$-scale radiative model [26] in which the loop contribution is genuine, i.e. self-protected like in the original Zee model. In its present variant the color singlet, weak triplet, hypercharge zero scalar field $\Delta \sim(1,3,0)$,

$$
\Delta=\frac{1}{\sqrt{2}} \sum_{j} \sigma_{j} \Delta^{j}=\left(\begin{array}{cc}
\frac{1}{\sqrt{2}} \Delta^{0} & \Delta^{+} \\
\Delta^{-} & -\frac{1}{\sqrt{2}} \Delta^{0}
\end{array}\right),
$$

is supplemented by a charged scalar singlet

$$
h^{+} \sim(1,1,1)
$$

and by additional three generations of vectorlike lepton doublets,

$$
\begin{aligned}
& E_{R} \equiv\left(E_{R}^{0}, E_{R}^{-}\right)^{T} \sim(1,2,-1 / 2), \\
& E_{L} \equiv\left(E_{L}^{0}, E_{L}^{-}\right)^{T} \sim(1,2,-1 / 2),
\end{aligned}
$$

which are needed to close the neutrino mass loop diagram displayed in Fig. 1. The corresponding vertices in the loop diagram are provided by Yukawa and quartic couplings in

$-\mathcal{L} \supset y_{1} \overline{\left(L_{L}\right)^{c}} E_{L} h^{+}+y_{2} \overline{L_{L}} \Delta E_{R}+\lambda_{7} H^{\dagger} \Delta H^{c} h^{+}+$H.c.

The vacuum expectation value $v_{\mathrm{SM}}$ of the SM Higgs doublet $H$ leads to the neutrino mass matrix

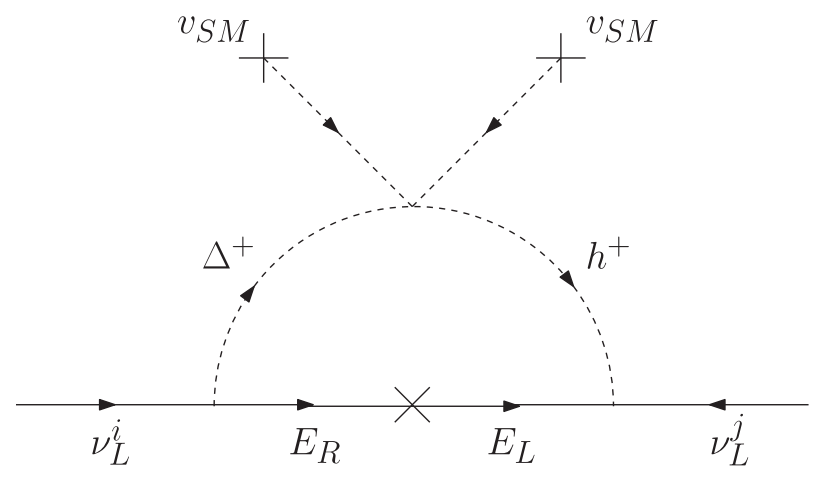

FIG. 1. The one-loop BPR [26] neutrino mass mechanism.

$$
\begin{aligned}
\mathcal{M}_{i j}= & \sum_{k=1}^{3} \frac{\left[\left(y_{1}\right)_{i k}\left(y_{2}\right)_{j k}+\left(y_{2}\right)_{i k}\left(y_{1}\right)_{j k}\right]}{8 \pi^{2}} \\
& \times \lambda_{7} v_{\mathrm{SM}}^{2} M_{E_{k}} f\left(M_{E_{k}}, m_{\Delta^{+}}, m_{h^{+}}\right),
\end{aligned}
$$

where $f\left(m_{1}, m_{2}, m_{3}\right)$ is a loop function specified in [26]. Assuming like in [26] the mass values $M_{E} \sim m_{\Delta^{+}} \sim$ $m_{h^{+}} \sim 200-500 \mathrm{GeV}$, Eq. (5) leads to $m_{\nu} \sim 0.1 \mathrm{eV}$ for the couplings $y_{1,2}$ and $\lambda_{7}$ of the order of $10^{-4}$. For definiteness, we will in most of this work keep masses of these new states fixed at $500 \mathrm{GeV}$. In principle, even much larger masses would lead to a viable neutrino mass model, with larger but still perturbative values of $y_{1,2}$ and $\lambda_{7}$. Still, as we shall discuss later, such scenarios would not bring much additional insight from the GUT perspective.

We display in Table I the SU(5) embedding of the SM extended by states in the neutrino mass model at hand. We note that additional potentially light BPR particles are described by the same SM group representations as those already populated by the SM states: new vectorlike fermionic doublets $E_{R, L}$ belong to the same representation as the Higgs $H^{c}$, and similarly for the charged scalar singlet $h^{+}$and the SM lepton singlet $e_{R}^{c}$, or the scalar adjoint triplet $\Delta$ and the spin one triplet $W_{\mu}^{i}$. Understanding the quantum numbers of SM particles was one of the main motivations which led to the development of GUTs. The fact that BPR states populate already established SM representations could be viewed as an additional motive to study them in the GUT setup.

\section{B. Matching BPR states with SU(5) irreps}

One of the strongest arguments in favor of the original Georgi-Glashow GUT scenario is a neat embedding of all SM fermion representations, with apparently arbitrary quantum numbers, into sum $\overline{\mathbf{5}}_{F} \oplus \mathbf{1 0}_{F}$ of just two complete lowest $\mathrm{SU}(5)$ representations. Since the gauge bosons have to belong to the adjoint multiplet of the SU(5) group, essentially the only remaining unknown has been the structure of the scalar sector. In the present study, this generalizes to the question of incorporating the well motivated BPR set of particles into the same GUT context. 
TABLE I. Particle content and the SU(5) embedding options of the BPR neutrino mass model [26].

\begin{tabular}{|c|c|c|}
\hline & $\mathrm{SM}+\mathrm{BPR}$ & $\subset S U(5)$ \\
\hline \multirow[t]{2}{*}{ Scalar } & $H=\left(1,2,+\frac{1}{2}\right)$ & $\mathbf{5}=\left(1,2,+\frac{1}{2}\right) \oplus\left(3,1,-\frac{1}{3}\right) ;$ or $\mathbf{4 5}, \mathbf{7 0}$ \\
\hline & $\begin{aligned} \Delta & =(1,3,0) \\
h^{+} & =(1,1,+1)\end{aligned}$ & $\begin{array}{c}\mathbf{2 4}=(1,3,0) \oplus(8,1,0) \oplus(1,1,0) \\
\oplus\left(3,2,-\frac{5}{6}\right) \oplus\left(\overline{3}, 2,+\frac{5}{6}\right) \\
\mathbf{1 0}=(1,1,+1) \oplus\left(\overline{3}, 1,-\frac{2}{3}\right) \oplus\left(3,2,+\frac{1}{6}\right)\end{array}$ \\
\hline \multirow[t]{2}{*}{ Fermion } & $\begin{aligned} 3 \times Q & =\left(3,2,+\frac{1}{6}\right) \\
3 \times u^{c} & =\left(\overline{3}, 1,-\frac{2}{3}\right) \\
3 \times e^{c} & =(1,1,+1) \\
3 \times L & =\left(1,2,-\frac{1}{2}\right) \\
3 \times d^{c} & =\left(\overline{3}, 1,+\frac{1}{3}\right)\end{aligned}$ & $3 \times \mathbf{1 0}=\left(3,2,+\frac{1}{6}\right) \oplus\left(\overline{3}, 1,-\frac{2}{3}\right) \oplus(1,1,+1)$ \\
\hline & $3 \times E_{R, L}=\left(1,2,-\frac{1}{2}\right)$ & $3 \times \overline{\mathbf{5}}=\left(1,2,-\frac{1}{2}\right) \oplus\left(\overline{3}, 1,+\frac{1}{3}\right) ;$ or $\overline{\mathbf{4 5}}, \overline{\mathbf{7 0}}$ \\
\hline Gauge & $\begin{array}{l}G_{\mu}=(8,1,0) \\
W_{\mu}=(1,3,0) \\
B_{\mu}=(1,1,0)\end{array}$ & $\mathbf{2 4}=(1,3,0) \oplus(8,1,0) \oplus(1,1,0) \oplus\left(3,2,-\frac{5}{6}\right) \oplus\left(\overline{3}, 2,+\frac{5}{6}\right.$ \\
\hline
\end{tabular}

Following the pattern of SM states, and general principles of economy and elegance, BPR states at hand may be expected to belong to the lowest possible representations of the $\mathrm{SU}(5)$ group. This would put scalar $\Delta$ in an adjoint $\mathbf{2 4}$, scalar $h^{+}$in $\mathbf{1 0}$, and vectorlike leptons $E_{R, L}$ in the appropriate number of $\mathbf{5} \oplus \overline{\mathbf{5}}$, which is a choice displayed in the right column of Table I. These new SU(5) irreps contain additional states displayed in Table I, which are not needed for the BPR neutrino mass mechanism. Some among these additional states will prove crucial in obtaining the desired gauge coupling unification.

To completely specify the structure of our model Lagrangian, we need to choose the SU(5) irreps that will contain the Standard Model Higgs $H$. Here the most economical choice would be $\mathbf{5}$, but, as we shall see, this would not lead to a viable GUT model. Thus, we will consider also the options where the Higgs state belongs to 45 or 70 irreps, ${ }^{1}$ and we complete $\mathrm{SU}(5) \supset \mathrm{SU}(3)_{c} \times$ $\mathrm{SU}(2)_{L} \times \mathrm{U}(1)_{Y}$ branching rules from Table I with

$$
\begin{aligned}
\mathbf{4 5}= & \left(1,2,+\frac{1}{2}\right) \oplus\left(3,1,-\frac{1}{3}\right) \oplus\left(3,3,-\frac{1}{3}\right) \oplus\left(\overline{3}, 1,+\frac{4}{3}\right) \oplus\left(\overline{3}, 2,-\frac{7}{6}\right) \\
& \oplus\left(\overline{6}, 1,-\frac{1}{3}\right) \oplus\left(8,2,+\frac{1}{2}\right) \\
\mathbf{7 0}= & \left(1,2,+\frac{1}{2}\right) \oplus\left(1,4,+\frac{1}{2}\right) \oplus\left(3,1,-\frac{1}{3}\right) \oplus\left(3,3,-\frac{1}{3}\right) \oplus\left(\overline{3}, 3,+\frac{4}{3}\right) \\
& \oplus\left(6,2,-\frac{7}{6}\right) \oplus\left(8,2,+\frac{1}{2}\right) \oplus\left(15,1,-\frac{1}{3}\right) .
\end{aligned}
$$

As is known, Higgs in a mixture of $\mathbf{5}$ and $\mathbf{4 5}$ can improve the GUT fermion mass relations, like in the GeorgiJarlskog mechanism [32]. However, in the present work we will not study the pattern of SM fermion masses.

On the other hand, a pattern of scalar masses and of new vectorlike lepton masses is important for our considerations, since these particles decisively affect the running of gauge couplings. We need then to check for any of these possibilities whether unification can be

\footnotetext{
${ }^{1}$ Using even higher SU(5) irreps would expose us to the danger of low Landau poles.
}

achieved for large enough $M_{\mathrm{GUT}}$, whether the scalar Lagrangian at the renormalizable level allows for required masses of particles and, finally, whether such a scenario is in compliance with phenomenological constraints and the general theoretical requirement of perturbativity [31,33].

\section{Gauge coupling unification criteria}

The unification of gauge couplings is controlled by the renormalization group equations (RGE) which govern the running of gauge couplings with the one-loop $\beta$ coefficients given by 


$$
\begin{aligned}
b_{i}= & -\frac{11}{3} \sum_{G} T\left(G_{i}\right) D\left(G_{i}\right)+\frac{4}{3} \sum_{F} T\left(F_{i}\right) D\left(F_{i}\right) \kappa \\
& +\frac{1}{3} \sum_{S} T\left(S_{i}\right) D\left(S_{i}\right) \eta .
\end{aligned}
$$

Here, the Dynkin indices $T\left(R_{i}\right)$ are defined as $T\left(R_{i}\right) \delta_{a b} \equiv$ $\operatorname{Tr}\left[\hat{T}_{a}\left(R_{i}\right) \hat{T}_{b}\left(R_{i}\right)\right]$ for generators $\hat{T}_{a}$ in gauge, fermion and scalar representations $G_{i}, F_{i}$ and $S_{i}$, respectively, and are conventionally normalized to $\frac{1}{2}$ for fundamental representations of $\mathrm{SU}(N)$ groups [and thus to $\frac{3}{5} \hat{Y}^{2}$ for $U(1)_{Y}$ ]. $D\left(R_{i}\right) \equiv \prod_{j \neq i} \operatorname{dim}\left(R_{j}\right), \kappa$ being $\frac{1}{2}$ (1) for Weyl (Dirac) fermions and $\eta$ being $\frac{1}{2}$ (1) for real (complex) scalars. The SM $\beta$ coefficients (including the Higgs doublet) are $b^{\mathrm{SM}}=\left(\frac{41}{10},-\frac{19}{6},-7\right)$, and RGE have the analytical solution

$$
\alpha_{i}^{-1}\left(M_{\mathrm{GUT}}\right)=\alpha_{i}^{-1}\left(m_{Z}\right)-\frac{1}{2 \pi} B_{i} \ln \frac{M_{\mathrm{GUT}}}{m_{\mathrm{Z}}},
$$

with coefficients

$$
B_{i}=b_{i}^{(\mathrm{SM})}+\sum_{m_{k}<M_{\mathrm{GUT}}} \Delta b_{i}^{(k)} r_{k},
$$

and the threshold weight factor of the BSM state $k$, defined as

$$
r_{k}=\frac{\ln M_{\mathrm{GUT}} / m_{k}}{\ln M_{\mathrm{GUT}} / m_{Z}},
$$

with a value between 1 (for $m_{k}=m_{Z}$ ) and 0 (for $\left.m_{k}=M_{\mathrm{GUT}}\right)$, depending on the mass of the BSM particle $m_{k}$. The sum in (10) goes over all BSM states, with $\Delta b_{i}^{(k)}=$ $b_{i}^{(k)}-b_{i}^{(k-1)}$ being the increase in the $\beta$ coefficients at the threshold of a given BSM state, and $b_{i}^{(0)}=b_{i}^{(\mathrm{SM})}$.

As first, the unification condition $\alpha_{1}\left(M_{\mathrm{GUT}}\right)=$ $\alpha_{2}\left(M_{\mathrm{GUT}}\right)=\alpha_{3}\left(M_{\mathrm{GUT}}\right) \equiv \alpha_{\mathrm{GUT}}$ can be expressed in the form of the so-called B-test [34,35]:

$$
\begin{aligned}
\frac{B_{23}}{B_{12}} & \equiv \frac{B_{2}-B_{3}}{B_{1}-B_{2}}=\frac{\alpha_{2}^{-1}\left(m_{Z}\right)-\alpha_{3}^{-1}\left(m_{Z}\right)}{\alpha_{1}^{-1}\left(m_{Z}\right)-\alpha_{2}^{-1}\left(m_{Z}\right)} \\
& =\frac{5}{8} \frac{\sin ^{2} \theta_{w}\left(m_{Z}\right)-\frac{\alpha_{\mathrm{EM}}\left(m_{Z}\right)}{\alpha_{3}\left(m_{Z}\right)}}{\frac{3}{8}-\sin ^{2} \theta_{w}\left(m_{Z}\right)}=0.718,
\end{aligned}
$$

where we used the average numerical values for the constants at $m_{Z}$ scale, as given in [36]. The comparison to the corresponding SM value 0.528 indicates that the couplings do not unify in the SM.
Second, the associated GUT scale

$$
\begin{aligned}
M_{\mathrm{GUT}} & =m_{Z} \exp \left(\frac{2 \pi\left(\alpha_{1}^{-1}\left(m_{Z}\right)-\alpha_{2}^{-1}\left(m_{Z}\right)\right)}{B_{1}-B_{2}}\right) \\
& =m_{Z} \exp \left(\frac{184.87}{B_{12}}\right)
\end{aligned}
$$

yields for the SM the value $M_{\mathrm{GUT}}=10^{13} \mathrm{GeV}$. Therefore, additional BSM states should improve unification and increase its scale up to at least $5 \times 10^{15} \mathrm{GeV}$ which is in agreement with proton lifetime bounds [37]. Such additional BSM states must therefore provide a negative net contribution to $B_{12}$ and positive to $B_{23}$.

\section{POSSIBLE GAUGE-UNIFICATION REALIZATIONS}

\section{A. Effect of BPR states on gauge unification}

Before embedding in the SU(5) GUT framework, we first investigate how the new states, needed for neutrino mass mechanism, influence the RGE running and to what extent they alone could satisfy the unification criteria from Sec. II C.

In Table II we list extra BPR states together with their contribution to pertinent combinations of $\beta$-function coefficients. As already stressed, the states with positive $B_{23}$ and negative $B_{12}$ are promising for unification. It can be readily seen that only $h^{+}$is not of this kind.

If we consider the default configuration with all BPR states close to the electroweak scale [i.e. weight factors from Eq. (11) being $r_{k} \sim 1$ ], one immediately observes that the B-test combination increases to $B_{23} / B_{12}=0.974$, from the SM value 0.528 , considerably overshooting the required value of 0.718 from Eq. (12). This is mostly due to the strong effect of three copies of BPR vectorlike lepton doublets. Since they actually double the RGE effect of previously mentioned six Higgs doublets, one can similarly achieve correct unification if they are set at the intermediate scale with the factor $r_{k} \sim 0.5$. However, again like in the six Higgs doublet case, the unification scale would be too low.

Indeed, one observes that there is no way to obtain high enough unification scale by using only BPR states. Namely, even if negative effects of the scalar $h^{+}$state are avoided by putting it at some very high scale, the total contribution of

TABLE II. Contributions of BPR states to RGE running, where threshold weights $r_{k}$ are defined in Eq. (11). Note that two Weyl fermion states $E_{L}$ and $E_{R}$ each come in $N_{\mathrm{vec}}=3$ copies.

\begin{tabular}{lccc}
\hline \hline$k$ & & $\Delta B_{23}$ & $\Delta B_{12}$ \\
\hline$h^{+}$ & $(1,1,1)$ & 0 & $\frac{1}{5} r_{k}$ \\
$\Delta$ & $(1,3,0)$ & $\frac{1}{3} r_{k}$ & $-\frac{1}{3} r_{k}$ \\
$E_{L, R}$ & $(1,2,-1 / 2)$ & $\frac{1}{3} r_{k}$ & $-\frac{2}{15} r_{k}$ \\
\hline \hline
\end{tabular}


$\Delta$ and $E_{L, R}$ states to $B_{12}$ is at most only $-17 / 15$ (for $r_{k}=1$ ) resulting in $M_{\mathrm{GUT}}=1.1 \times 10^{15} \mathrm{GeV}$ as the maximal possible GUT scale, even if one would completely disregard the condition of the gauge coupling crossing.

In conclusion, BPR states alone cannot lead to a successful unification that would at the same time respect proton decay bounds. To achieve that, some other states below the GUT scale should be invoked. Such states are naturally provided by embedding the BPR model in a SU (5) unification framework, as we shall show in detail.

\section{B. Higgs doublet in $\mathbf{5}_{\boldsymbol{H}}$}

As explained in Sec. II B, we will first try the simplest possible $\mathrm{SU}(5)$ embedding of the BPR mechanism, where the SM Higgs doublet becomes a member of the $\mathbf{5}_{H}$. The scalar sector of this model contains $\mathbf{5}_{H}, \mathbf{1 0}_{S}$ and $\mathbf{2 4}_{S}$ multiplets [we use subscript $H$ on those scalar irreps of SU(5) which contain the SM Higgs field], and there are $N_{\text {vec }}$ generations of vectorlike matter in $\mathbf{5}_{F} \oplus \overline{\mathbf{5}}_{F}$, on top of the Standard Model quarks and leptons in $n_{g}=3$ generations of $\mathbf{1 0}_{F}$ and $\overline{\mathbf{5}}_{F}$, and gauge bosons in the adjoint $\mathbf{2 4}_{g}$ representation, as displayed in Table I. Their contributions to the RGE running are listed in Table III.

It is known that the simplest Georgi-Glashow SU(5) GUT suffers from the doublet-triplet splitting problem, where leptoquark $S_{1}=(3,1,-1 / 3)$, which completes $\mathbf{5}_{H}$ together with SM Higgs, has to be much heavier than the Higgs so that it does not induce the fast proton decay. There is nothing preventing the other scalar $\mathrm{SU}(5)$ multiplets to be

TABLE III. BSM contributions to RGE running in the simplest $\mathrm{SU}(5)$ embedding of the BPR mechanism. $H$ stands for SM Higgs doublet whose contribution has already been accounted for by $b_{i}^{(\mathrm{SM})}$. The massless scalar leptoquarks $X$ and $Y$ get absorbed into longitudinal components of massive gauge bosons, as dictated by the Nambu-Goldstone mechanism - the $\beta$ coefficients of these scalars thus enter at the same scale as heavy vectors (i.e. $r_{k} \approx 0$ ).

\begin{tabular}{lcccc}
\hline \hline & $k$ & & $\Delta B_{23}$ & $\Delta B_{12}$ \\
\hline$H$ & $(1,2,1 / 2)$ & $\mathbf{5}_{H}$ & $\frac{1}{6} r_{k}$ & $-\frac{1}{15} r_{k}$ \\
$S_{1}$ & $(3,1,-1 / 3)$ & & $-\frac{1}{6} r_{k}$ & $\frac{1}{15} r_{k}$ \\
$h^{+}$ & $(1,1,1)$ & $\mathbf{1 0}_{S}$ & 0 & $\frac{1}{5} r_{k}$ \\
& $(\overline{3}, 1,-2 / 3)$ & & $-\frac{1}{6} r_{k}$ & $\frac{4}{15} r_{k}$ \\
& $(3,2,1 / 6)$ & & $\frac{1}{6} r_{k}$ & $-\frac{7}{15} r_{k}$ \\
& $(1,1,0)$ & $\mathbf{2 4}_{S}$ & 0 & 0 \\
$\Delta$ & $(1,3,0)$ & & $\frac{1}{3} r_{k}$ & $-\frac{1}{3} r_{k}$ \\
& $(8,1,0)$ & & $-\frac{1}{2} r_{k}$ & 0 \\
$X, Y$ & $(3,2,-5 / 6)$ & & $\frac{1}{12} r_{k}$ & $\frac{1}{6} r_{k}$ \\
$\bar{X}, \bar{Y}$ & $(\overline{3}, 2,5 / 6)$ & & $\frac{1}{12} r_{k}$ & $\frac{1}{6} r_{k}$ \\
$E_{L, R}$ & $(1,2,-1 / 2)$ & $\overline{5}_{F}$ & $\frac{1}{3} r_{k}$ & $-\frac{2}{15} r_{k}$ \\
& $(\overline{3}, 1,1 / 3)$ & & $-\frac{1}{3} r_{k}$ & $\frac{2}{15} r_{k}$ \\
\hline \hline
\end{tabular}

split, and we will check in Sec. IV that our splitting patterns are consistent with the structure of the general scalar potential. Still, whatever the actual mechanism responsible for the multiplet splitting, there is no reason to assume that this mechanism is somehow aligned with the neutrino mass mechanism. Thus, we will be quite general in allowing the splitting of masses within SU(5) multiplets.

With this freedom, and having at our disposal a variety of states from Table III with different RGE behavior, the unification prospects look promising. Indeed, we have found several scenarios where coupling constants correctly unify (cross at the single point). However, we also find that, whatever the masses of BSM states between $M_{Z}$ and $M_{\mathrm{GUT}}$, the highest possible unification scale in this model is $M_{\mathrm{GUT}}<10^{15} \mathrm{GeV}$, in violation of experimental bounds on proton decay widths. Thus, this simplest embedding of the proposed neutrino mass model with the Higgs doublet restricted to $\mathbf{5}_{H}$ irrep is ruled out.

\section{Higgs doublet in $\mathbf{4 5}_{H}$}

Next we consider the scenario where the SM Higgs doublet is embedded into $\mathbf{4 5}_{H}$ instead of $\mathbf{5}_{H}$, or in some mixture of both. The larger particle content can help in raising the unification scale and, as a bonus, this setup can serve to correct the wrong mass relations between charged leptons and down-type quarks at the renormalizable level which are usually obtained in the simplest $\mathrm{SU}(5)$ models. The $\beta$ coefficients of the extra states from scalar $\mathbf{4 5}_{H}$ can be found in Table IV, which should be added to states in Table III to obtain a complete embedding of the SM Higgs and the BPR states into SU(5) multiplets.

In this more realistic model one finds many ways in which one can achieve a correct unification, so we need to specify some criteria that will lead to a set of models covering all interesting scenarios; let us list those implemented in our study.

(i) First, note that if all states of a given SU(5) irrep appear at the same mass scale, their effect on RGE cancels [contributions to either $B_{23}$ or $B_{12}$ from all

TABLE IV. Contributions of $\mathbf{4 5}_{H}$ to running. For a complete model the multiplets from Table III are to be added. The states $S_{1}$, $S_{3}$ and $\tilde{S}_{1}$ are leptoquarks that, if light, would induce too fast proton decay.

\begin{tabular}{|c|c|c|c|c|}
\hline & $k$ & & $\Delta B_{23}$ & $\Delta B_{12}$ \\
\hline$\Sigma_{a}$ & $(1,2,1 / 2)$ & & $\frac{1}{6} r_{k}$ & $-\frac{1}{15} r_{k}$ \\
\hline$S_{1} \equiv \Sigma_{b}$ & $(3,1,-1 / 3)$ & & $-\frac{1}{6} r_{k}$ & $\frac{1}{15} r$ \\
\hline$S_{3} \equiv \Sigma_{c}$ & $(3,3,-1 / 3)$ & & $3^{\circ}$ & \\
\hline$\tilde{S}_{1} \equiv \Sigma_{d}$ & $(\overline{3}, 1,4 / 3)$ & $\mathbf{4 5}_{H}$ & & \\
\hline$\Sigma_{e}$ & $(\overline{3}, 2,-7 / 6)$ & & $\frac{1}{6} r_{k}$ & \\
\hline$\Sigma_{f}$ & $(\overline{6}, 1,-1 / 3)$ & & $-\frac{5}{6} r_{k}$ & $\frac{2}{15} r_{k}$ \\
\hline$\Sigma_{g}$ & $(8,2,1 / 2)$ & & $-\frac{2}{3} r_{k}$ & $-\frac{8}{15} r_{k}$ \\
\hline
\end{tabular}


states of a given SU(5) irrep in, e.g., Table III, taking the same $r_{k}$, add up to zero]. Thus, we will fix the BPR states close to the electroweak scale (for definiteness, we put them at $500 \mathrm{GeV}$ ), and by doing so we do not lose much generality, from the standpoint of RGE, because the effect on unification of making e.g. BPR vectorlike leptons $E_{L, R}$ heavier is the same as making the rest of the multiplet [in this case $(\overline{3}, 1,1 / 3)$ states] lighter. (Some generality may be lost if some of these other states cannot be made lighter for other reasons.)

(ii) Next, since, as discussed before, we allow general splitting of SU(5) multiplets, with any experimentally allowed mass for the rest of BSM states (see Sec. IV), we have enough freedom to achieve the exact gauge coupling unification i.e. a fulfillment of the B-test, see Eq. (12). Then, we require the GUT scale $M_{\mathrm{GUT}}$ larger than $2 \times 10^{15} \mathrm{GeV}$. The lowest experimental bound, coming from proton decay searches, is actually about $5 \times 10^{15}$; however, it turns out that for most of the scenarios presented here, a simplified analysis (ignoring the Yukawa contributions to twoloop RGE) shows that improving RGE to two-loop accuracy increases $M_{\mathrm{GUT}}$ beyond $5 \times 10^{15} \mathrm{GeV}$.

(iii) We will also exclude scenarios with very heavy new BSM particles, with masses between $\sim 10^{11} \mathrm{GeV}$ and $M_{\mathrm{GUT}}$. Otherwise, one can always take any successful model, add some particles slightly below $M_{\mathrm{GUT}}$ that will have only small influence on running, and thus obtain many more models which will be qualitatively the same as the ones presented in this paper, only more complicated. This requirement at the same time excludes from consideration leptoquarks $S_{1}=(3,1,-1 / 3), \tilde{S}_{1}=(\overline{3}, 1,4 / 3)$ and $S_{3}=(3,3,-1 / 3)$ which, if lighter than $\sim 10^{11} \mathrm{GeV}$ would naturally lead to proton decay in violation of experimental limits.

(iv) For all BSM particles we take $500 \mathrm{GeV}$ as a lower bound on their masses. Direct LHC searches sometimes put higher bounds on such states, but these bounds are often obtained only within specific benchmark scenarios. For example, the recent CMS search [38] puts the lower bound of $3 \mathrm{TeV}$ on the color octet state, like $(8,1,0)$ from $\mathbf{2 4}_{S}$, but only within the benchmark model of Refs. [39,40], where couplings to loop fermions in production and decay are taken to be of order one (see also Ref. [41]).

(v) We include only particles from single copies of scalar SU(5) irreps $\mathbf{5}_{H}, \mathbf{1 0}_{S}, \mathbf{2 4}_{S}$ and $\mathbf{4 5}_{H}$ (or, in the next section $\mathbf{7 0}_{H}$ ) and $N_{\text {vec }}=n_{\mathrm{g}}=3$ copies of vectorlike $\overline{\mathbf{5}}_{F}$, which are all already needed for embedding the BPR neutrino mass mechanism.

Under these conditions, we performed the exhaustive search of the parameter space, using the algorithm specified in Appendix A, and resulting in successful scenarios listed in Table V. As explained in Appendix A, when a given set
TABLE V. Seven unification scenarios with SM Higgs in $\mathbf{5}$ and/or 45 of SU(5) and BPR states fixed at $\sim 0.5 \mathrm{TeV}$.

\begin{tabular}{|c|c|c|c|c|c|c|c|c|}
\hline \multicolumn{2}{|l|}{ irreps } & \multicolumn{7}{|c|}{$m_{k}[\mathrm{TeV}]$} \\
\hline SM & SU(5) & A1 & A2 & A3 & A4 & A5 & A6 & A7 \\
\hline$(\overline{3}, 1,1 / 3)$ & $\overline{\mathbf{5}}_{F}$ & 5000 & & $2.3 \times 10^{6}$ & 450 & & $2 \times 10^{5}$ & \\
\hline $\begin{array}{l}(\overline{3}, 1,-2 / 3) \\
(3,2,1 / 6)\end{array}$ & $\mathbf{1 0}_{S}$ & 0.5 & & 0.5 & 0.5 & & 0.5 & $\begin{array}{l}2.4 \\
0.5\end{array}$ \\
\hline$(8,1,0)$ & $\mathbf{2 4}_{S}$ & & 0.5 & 0.5 & & 0.5 & 0.5 & \\
\hline $\begin{array}{l}(1,2,1 / 2) \\
(\overline{6}, 1,-1 / 3)\end{array}$ & $\mathbf{4 5} \mathbf{5}_{H}$ & & 90 & & 0.5 & $\begin{array}{l}260 \\
0.5\end{array}$ & 0.5 & 0.5 \\
\hline$(8,2,1 / 2)$ & & 0.5 & 0.5 & 0.5 & 0.5 & 0.5 & 0.5 & 0.5 \\
\hline$M_{\mathrm{GUT}}^{\max } /\left(10^{15}\right.$ & $\mathrm{GeV})$ & 2.8 & 2.5 & 6.2 & 2.8 & 2.8 & 6.2 & 6.5 \\
\hline
\end{tabular}

of new BSM states offers a continuum of possible GUT scenarios (with different spectra), we represent this continuum by a specific choice of spectrum with the minimal average mass of particles. ${ }^{2}$ Such a choice is motivated, besides the need for definiteness, by the desire to focus on models which have maximal discovery potential at LHC and future colliders.

Note that a light $(8,2,1 / 2)$ is the only other allowed representation in $45_{H}$ with a negative $B_{12}$ contribution needed to increase $M_{\mathrm{GUT}}$ and thus suppressing the proton decay. Of course by itself it does not help the unification due to negative $B_{23}$ (acting alone it can decrease $B_{23} / B_{12}$ to 0.470 ), but its strong effect on unification scale is important for all models displayed in Table V.

\section{Higgs doublet in $\mathbf{7 0}_{H}$}

If we opt for SM Higgs belonging to $\mathbf{7 0}_{H}$ instead of $\mathbf{4 5}_{H}$ (in addition to $\mathbf{5}_{H}$ ), the search proceeds under the conditions explicated in the previous subsection, and the $\beta$ coefficients of the extra states can be found in Table VI.

In this setup, we find three unification scenarios displayed in Table VII to which scenarios A1, A3, A4 and A6 from Table V should be added, since they employ only states from $\mathbf{4 5}_{H}$ that are also present in $\mathbf{7 0}_{H}$. From this search we have explicitly excluded representation $(15,1,-1 / 3)$ which, if light, leads to Landau poles below $M_{\mathrm{GUT}}$. To avoid this, it should be heavier than at least $10^{7} \mathrm{GeV}$ [31] so that its effect on the RG running would be diminished. Including also this representation leads to 16 additional scenarios beyond those in Tables V and VII, which we have chosen not to list.

Interestingly, all viable scenarios in this setup, as displayed in Table VII, involve the color triplet $(\overline{3}, 1,1 / 3)$ at the same scale $(500 \mathrm{GeV})$ as BPR vectorlike leptons, making the irrep $\overline{\mathbf{5}}_{F}$ complete and nullifying its influence on the RGE running. Thus, these states can all be at any

\footnotetext{
${ }^{2}$ More precisely, maximal average threshold weight factor $r_{k}$ defined in Eq. (11).
} 
TABLE VI. Contributions of $\mathbf{7 0}_{H}$ to RGE running. For a complete model, the multiplets from Table III are to be added. The states $S_{1}$ and $S_{3}$ are leptoquarks that, if light, would naturally induce too fast proton decay.

\begin{tabular}{|c|c|c|c|c|}
\hline & $k$ & & $\Delta B_{23}$ & $\Delta B_{12}$ \\
\hline$\Omega_{a}$ & $(1,2,1 / 2)$ & & $\frac{1}{6} r_{k}$ & $-\frac{1}{15} r_{k}$ \\
\hline$S_{1} \equiv \Omega_{b}$ & $(3,1,-1 / 3)$ & & $-\frac{1}{6} r_{k}$ & $\frac{1}{15} r_{k}$ \\
\hline$S_{3} \equiv \Omega_{c}$ & $(3,3,-1 / 3)$ & & $\frac{3}{2} r_{k}$ & $-\frac{9}{5} r_{k}$ \\
\hline$\Omega_{d}$ & $(\overline{3}, 3,4 / 3)$ & & & \\
\hline$\Omega_{e}$ & $(6,2,-7 / 6)$ & $\mathbf{7 0}_{H}$ & & $\frac{34}{15} r_{k}$ \\
\hline$\Omega_{f}$ & $(15,1,-1 / 3)$ & & $-\frac{10}{3} r_{k}$ & $\frac{1}{3} r_{k}$ \\
\hline$\Omega_{g}$ & $(8,2,1 / 2)$ & & $-\frac{2}{3} r_{k}$ & $-\frac{8}{15} r_{k}$ \\
\hline$\Omega_{h}$ & $(1,4,1 / 2)$ & & $\frac{5}{3} r_{k}$ & $-\frac{22}{15} r_{k}$ \\
\hline
\end{tabular}

TABLE VII. Three unification scenarios with SM Higgs in $\mathbf{5}_{H}$ and $\mathbf{7 0}_{H}$ of $\mathrm{SU}(5)$, and BPR states fixed at $\sim 0.5 \mathrm{TeV}$. These are at the same time all viable scenarios under assumption of unsplit vectorlike fermion $\mathbf{5}_{F}$.

\begin{tabular}{lccccc}
\hline \hline irreps & & & \multicolumn{3}{c}{$m_{k}[\mathrm{TeV}]$} \\
\cline { 1 - 2 } \cline { 4 - 6 } $\mathrm{SM}$ & $\mathrm{SU}(5)$ & & $\mathrm{B} 1$ & $\mathrm{~B} 2$ & $\mathrm{~B} 3$ \\
\hline$(\overline{3}, 1,1 / 3)$ & $\overline{\mathbf{5}}_{F}$ & & 0.5 & 0.5 & 0.5 \\
$(3,2,1 / 6)$ & $\mathbf{1 0}_{S}$ & & & 0.5 \\
$(8,1,0)$ & $\mathbf{2 4}_{S}$ & & 0.5 & 0.5 \\
$(1,4,1 / 2)$ & $\mathbf{7 0}_{H}$ & $1.8 \times 10^{6}$ & $1.3 \times 10^{4}$ & $6.3 \times 10^{6}$ \\
$(8,2,1 / 2)$ & & 0.5 & 0.5 & 0.5 \\
$M_{\mathrm{GUT}}^{\max } /\left(10^{15} \mathrm{GeV}\right)$ & & 2.6 & 10.6 & 32.0 \\
\hline \hline
\end{tabular}

other scale as well, without changing the gauge unification property of the model. We note that there are no viable scenarios with such unsplit fermion $\mathbf{5}_{F}$ and with Higgs in $\mathbf{4 5}_{H}$, i.e. in the framework of Sec. III C.

\section{SCALAR POTENTIAL AND SPECTRUM}

In Secs. III C and III D we singled out viable scenarios in two variants of nonsupersymmetric $\mathrm{SU}(5)$ unification. Now we are presenting for them the relevant expressions for the scalar potentials and the resulting mass spectra, demonstrating their consistency with the appropriate scalar sector extensions. The scalar sector for the scenarios from Sec. III C contains

(i) $\mathbf{2 4}_{S}$ : an adjoint 24-dimensional real traceless representation $\chi_{j}^{i}$;

(ii) $\mathbf{5}_{H}$ : a fundamental five-dimensional complex representation $H^{i}$;

(iii) $\mathbf{1 0}_{S}$ : an antisymmetric ten-dimensional complex representation $\phi^{i j}$

(iv) $\mathbf{4 5}_{H}$ : a 45 -dimensional complex two-index antisymmetric traceless representation $\Sigma_{k}^{i j}$,

which get decomposed under the SM gauge group as displayed in Table I and Eq. (6). For the scenario from Sec. III D, $\Sigma_{k}^{i j}$ is replaced with

(iv) $\mathbf{7 0}_{H}$ : a 70-dimensional complex two-index symmetric traceless representation $\Omega_{k}^{i j}$,

with the SM decomposition displayed in Eq. (7). The details of individual representations can be found in Appendix B. The following fields from this set can develop potentially nonvanishing VEVs:

(i) the SM singlet field from $\chi_{j}^{i}$ whose GUT scale VEV $\left\langle(1,1,0)_{\chi}\right\rangle \equiv v_{\mathrm{GUT}}$ results in breaking $S U(5) \rightarrow$ $S U(3)_{c} \times S U(2)_{L} \times U(1)_{Y}$

(ii) the neutral components of the weak doublets from $H^{i}$ and $\Sigma_{k}^{i j}$ whose $S U(2)_{L} \times U(1)_{Y} \rightarrow U(1)_{Q}$ breaking $\operatorname{VEVs}\left\langle\left(1,2,+\frac{1}{2}\right)_{H}\right\rangle \equiv v_{5}$ and $\left\langle\left(1,2,+\frac{1}{2}\right)_{\Sigma}\right\rangle \equiv$ $v_{45}$ are subject to the condition $v_{5}^{2}+v_{45}^{2}=v_{\mathrm{SM}}^{2}$;

(iii) the neutral components of $\left(1,2,+\frac{1}{2}\right)_{\Omega}$ and $\left(1,4,+\frac{1}{2}\right)_{\Omega}$ from $\Omega_{k}^{i j}$ can also develop VEVs of the order of electroweak scale;

(iv) the neutral component of the weak triplet from $\chi_{j}^{i}$ can develop a tiny (few $\left.\mathrm{GeV}\right) \operatorname{VEV}\left\langle(1,3,0)_{\chi}\right\rangle$ severely constrained by the measured electroweak precision $\rho$ parameter.

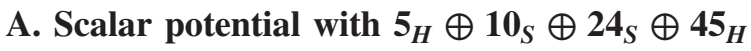

We are only interested in the part of the renormalizable scalar potential that provides the $\mathrm{SU}(3)_{c} \times \mathrm{SU}(2)_{L} \times$ $\mathrm{U}(1)_{Y}$ invariant contributions to the scalar spectrum proportional to $v_{\mathrm{GUT}}$ :

$$
V=V_{24}(\chi)+V_{5}(H, \chi)+V_{10}(\phi, \chi)+V_{45}(\Sigma, \chi)+V_{\text {mix }}(H, \chi, \Sigma) .
$$

Here

$$
\begin{gathered}
V_{24}=-\frac{1}{2} m_{\chi}^{2} \chi_{j}^{i} \chi_{i}^{j}+\sqrt{\frac{10}{3}} \mu_{\chi} \chi_{j}^{i} \chi_{k}^{j} \chi_{i}^{k}+\frac{1}{8} \lambda_{1} \chi_{j}^{i} \chi_{i}^{j} \chi_{l}^{k} \chi_{k}^{l}+\frac{15}{2} \lambda_{2} \chi_{j}^{i} \chi_{k}^{j} \chi_{l}^{k} \chi_{i}^{l}, \\
V_{5}=m_{H}^{2} H_{i}^{*} H^{i}+\sqrt{30} \mu_{H} H_{i}^{*} \chi_{j}^{i} H^{j}+\alpha_{1} H_{i}^{*} H^{i} \chi_{k}^{j} \chi_{j}^{k}+30 \alpha_{2} H_{i}^{*} \chi_{j}^{i} \chi_{k}^{j} H^{k}, \\
V_{10}=-m_{\phi}^{2} \phi_{i j}^{*} \phi^{j i}+2 \sqrt{30} \mu_{\phi} \phi_{i j}^{*} \chi_{k}^{j} \phi^{k i}+\beta_{1} \phi_{i j}^{*} \phi^{j i} \chi_{l}^{k} \chi_{k}^{l}+60 \beta_{2} \phi_{i j}^{*} \chi_{k}^{j} \chi_{l}^{k} \phi^{l i}+30 \beta_{3} \phi_{i j}^{*} \chi_{k}^{j} \phi^{k l} \chi_{l}^{i},
\end{gathered}
$$




$$
\begin{aligned}
V_{45}= & m_{\Sigma}^{2} \Sigma_{k}^{i j} \Sigma_{i j}^{* k}+4 \sqrt{30} \mu_{\Sigma} \Sigma_{k}^{i j} \Sigma_{i j}^{* l} \chi_{l}^{k}+8 \sqrt{30} \mu_{\Sigma}^{\prime} \Sigma_{k}^{i j} \Sigma_{i l}^{* k} \chi_{j}^{l} \\
& +\eta_{1} \Sigma_{k}^{i j} \Sigma_{i j}^{* k} \chi_{m}^{l} \chi_{l}^{m}+120 \eta_{2} \Sigma_{k}^{i j} \Sigma_{i j}^{* l} \chi_{m}^{k} \chi_{l}^{m}+240 \eta_{3} \Sigma_{k}^{i j} \Sigma_{i l}^{* k} \chi_{m}^{l} \chi_{j}^{m} \\
& +\frac{48}{5} \eta_{4} \Sigma_{k}^{i j} \Sigma_{i m}^{* l} \chi_{j}^{k} \chi_{l}^{m}+120 \eta_{5} \Sigma_{k}^{i j} \Sigma_{i m}^{* l} \chi_{j}^{m} \chi_{l}^{k}+120 \eta_{6} \Sigma_{k}^{i j} \Sigma_{l m}^{* k} \chi_{i}^{l} \chi_{j}^{m}, \\
V_{\text {mix }}= & \frac{12 \sqrt{5}}{5} \tau \Sigma_{k}^{i j} \chi_{i}^{k} H_{j}^{*}+12 \sqrt{2} \kappa_{1} \Sigma_{k}^{i j} \chi_{i}^{k} \chi_{j}^{l} H_{l}^{*}+12 \sqrt{2} \kappa_{2} \Sigma_{k}^{i j} \chi_{l}^{k} \chi_{i}^{l} H_{j}^{*}+\text { H.c. },
\end{aligned}
$$

and the summation over one upper and one lower repeating index is assumed. The potential contains nine real parameters $\left\{m_{\chi}, \mu_{\chi}, m_{H}, \mu_{H}, m_{\phi}, \mu_{\phi}, m_{\Sigma}, \mu_{\Sigma}, \mu_{\Sigma}^{\prime}\right\}$ and one complex parameter $\{\tau\}$ with positive dimension of mass. There are an additional thirteen real and two complex dimensionless parameters $\left\{\lambda_{1}, \lambda_{2}, \alpha_{1}, \alpha_{2}, \beta_{1}, \beta_{2}, \beta_{3}, \eta_{1}, \eta_{2}, \eta_{3}, \eta_{4}, \eta_{5}, \eta_{6}\right\}$ and $\left\{\kappa_{1}, \kappa_{2}\right\}$, respectively. The signs and various symmetry factors are introduced for convenience. Note that in the unbroken phase the mass terms $\left\{-m_{\chi}^{2}, m_{H}^{2}, m_{\phi}^{2}, m_{\Sigma}^{2}\right\}$ represent the squared masses of the corresponding $\mathrm{SU}(5)$ representations (with the conventional prefactor $\frac{1}{2}$ for the real scalar fields and 1 for complex scalars).

The spectrum presented in Tables VIII and IX is computed in the minimum of the scalar potential (the vacuum state) obtained for

$$
\frac{\partial\langle V\rangle}{\partial v_{\mathrm{GUT}}} \equiv 0,
$$

where

$$
m_{\chi}^{2}=\frac{1}{2} v_{\mathrm{GUT}}\left(2 \mu_{\chi}+\left(\lambda_{1}+14 \lambda_{2}\right) v_{\mathrm{GUT}}\right),
$$

TABLE VIII. The scalar spectrum for the simplest SU(5) embedding of BPR model with only $\mathbf{5}_{H}, \mathbf{1 0}_{S}$ and $\mathbf{2 4}_{S}$ multiplets, which corresponds to setting to zero the parameters in $V_{45}$ and $V_{\text {mix }}$. Their masses remain unchanged even after adding $\mathbf{4 5}_{H}$ or $\mathbf{7 0}_{H}$ to the particle content. Note that the parameters $\mu_{H}, \mu_{\phi}$ and $\mu_{\chi}$ should be understood as multiplied by $v_{\mathrm{GUT}}$ and $\alpha_{1}, \alpha_{2}, \beta_{1}, \beta_{2}, \beta_{3}$, $\lambda_{1}$ and $\lambda_{2}$ by $v_{\text {GUT }}^{2}$, while each of the masses is a sum of the pertinent contributions. For example, $m^{2}(3,1,-1 / 3)_{H}=$ $m_{H}^{2}-2 \mu_{H} v_{\mathrm{GUT}}+\left(\alpha_{1}+4 \alpha_{2}\right) v_{\mathrm{GUT}}^{2}$.

\begin{tabular}{lccccccccccccc}
\hline \hline & $m_{H}^{2}$ & $\mu_{H}$ & $\alpha_{1}$ & $\alpha_{2}$ & $m_{\phi}^{2}$ & $\mu_{\phi}$ & $\beta_{1}$ & $\beta_{2}$ & $\beta_{3}$ & $\mu_{\chi}$ & $\lambda_{1}$ & $\lambda_{2}$ \\
\hline$m^{2}\left(1,2,+\frac{1}{2}\right)_{H}$ & 1 & 3 & 1 & 9 & & & & & & & & \\
$m^{2}\left(3,1,-\frac{1}{3}\right)_{H}$ & 1 & -2 & 1 & 4 & & & & & & & & \\
$m^{2}\left(3,2,+\frac{1}{6}\right)_{\phi}$ & & & & & 1 & -1 & -1 & -13 & 6 & & & \\
$m^{2}\left(\overline{3}, 1,-\frac{2}{3}\right)_{\phi}$ & & & & 1 & 4 & -1 & -8 & -4 & & \\
$m^{2}(1,1,+1)_{\phi}$ & & & & 1 & -6 & -1 & -18 & -9 & & & \\
$m^{2}(1,1,0)_{\chi}$ & & & & & & & & & 1 & 1 & 14 \\
$m^{2}(1,3,0)_{\chi}$ & & & & & & & & & 5 & & 20 \\
$m^{2}(8,1,0)_{\chi}$ & & & & & & & & -5 & 5 \\
\hline \hline
\end{tabular}

and $v_{\mathrm{GUT}}$ is kept as a free parameter. The six massless complex scalar states $\left(3,2,-\frac{5}{6}\right)_{\chi}$ are absorbed into longitudinal components of twelve heavy gauge bosons.

The two $\left(1,2,+\frac{1}{2}\right)$ representations from $\mathbf{5}_{H}$ and $\mathbf{4 5}_{H}$ mix to form a SM Higgs doublet responsible for electroweak symmetry breaking. To compute their physical masses one needs to diagonalize the matrix

$$
\left(\begin{array}{cc}
m^{2}\left(1,2,+\frac{1}{2}\right)_{H} & m^{2}\left(1,2,+\frac{1}{2}\right)_{\text {mix }} \\
\left(m^{2}\left(1,2,+\frac{1}{2}\right)_{\text {mix }}\right)^{*} & m^{2}\left(1,2,+\frac{1}{2}\right)_{\Sigma}
\end{array}\right) .
$$

A similar diagonalization proceeds for the states $\left(3,1,-\frac{1}{3}\right)$ from $H$ and $\Sigma$. One of the masses needs to be around the weak scale to correspond to the SM Higgs. It can as well be fine-tuned to zero, since in our case we have neglected all the $v_{\text {SM }}$ contributions to spectrum.

When both of the Higgs doublets develop a nonvanishing VEV the Georgi-Jarlskog mechanism can be implemented to account for the observed masses of light fermions. It is also interesting to note that by excluding the mixing terms ( $V_{\text {mix }}$ with coefficients $\tau, \kappa_{1}$ and $\kappa_{2}$ ), as for example in the scenario without $\mathbf{5}_{H}$ where the Higgs doublet belongs entirely to $\mathbf{4 5}_{H}$, the masses of fields from $\Sigma$ are not linearly independent, and the following relation among them holds:

$$
\begin{aligned}
& m^{2}\left(1,2,+\frac{1}{2}\right)_{\Sigma}-\frac{3}{4} m^{2}\left(3,1,-\frac{1}{3}\right)_{\Sigma}-\frac{9}{8} m^{2}\left(3,3,-\frac{1}{3}\right)_{\Sigma} \\
& \quad-\frac{3}{4} m^{2}\left(\overline{3}, 1,+\frac{4}{3}\right)_{\Sigma}+\frac{3}{4} m^{2}\left(\overline{3}, 2,-\frac{7}{6}\right)_{\Sigma} \\
& \quad-\frac{3}{8} m^{2}\left(\overline{6}, 1,-\frac{1}{3}\right)_{\Sigma}+\frac{5}{4} m^{2}\left(8,2,+\frac{1}{2}\right)_{\Sigma}=0
\end{aligned}
$$

However, in the most general case the above expressions for scalar masses are all linearly independent. One can simplify the spectrum even further by imposing an additional $\mathbb{Z}_{2}$ symmetry under which in Eq. (15) $\mu_{\chi} \rightarrow 0$, thus imposing a strong correlation between the weak triplet and the color octet masses:

$$
\begin{gathered}
m^{2}(1,3,0)_{\chi}=20 \lambda_{2} v_{\mathrm{GUT}}^{2}=4 m^{2}(8,1,0)_{\chi}, \\
m^{2}(1,1,0)_{\chi}=2 m_{\chi}^{2} .
\end{gathered}
$$


TABLE IX. Additional contribution to the scalar spectrum in the vacuum after adding $\mathbf{4 5}_{H}$ to the $\mathbf{5}_{H} \oplus \mathbf{1 0}_{S} \oplus \mathbf{2 4}_{S}$ model. The last two rows represent the mixing between $\mathbf{5}_{H}$ and $\mathbf{4 5}_{H}$. Again, the parameters $\mu_{\Sigma}, \mu_{\Sigma}^{\prime}$ and $\tau$ should be multiplied by $v_{\mathrm{GUT}}$ and $\eta_{1}, \eta_{2}, \eta_{3}, \eta_{4}, \eta_{5}, \eta_{6}, \kappa_{1}$ and $\kappa_{2}$ by $v_{\mathrm{GUT}}^{2}$.

\begin{tabular}{|c|c|c|c|c|c|c|c|c|c|c|c|c|}
\hline & $m_{\Sigma}^{2}$ & $\mu_{\Sigma}$ & $\mu_{\Sigma}^{\prime}$ & $\eta_{1}$ & $\eta_{2}$ & $\eta_{3}$ & $\eta_{4}$ & $\eta_{5}$ & $\eta_{6}$ & $\tau$ & $\kappa_{1}$ & $\kappa_{2}$ \\
\hline$m^{2}\left(1,2,+\frac{1}{2}\right)_{\Sigma}$ & 1 & 7 & 19 & 1 & 31 & 67 & 3 & 26 & 21 & & & \\
\hline$m^{2}\left(3,1,-\frac{1}{3}\right)_{\Sigma}$ & 1 & 2 & -6 & 1 & 26 & 42 & 4 & 11 & -4 & & & \\
\hline$m^{2}\left(3,3,-\frac{1}{3}\right)_{\Sigma}$ & 1 & 12 & 4 & 1 & 36 & 52 & & 6 & -24 & & & \\
\hline$m^{2}\left(\overline{3}, 1,+\frac{4}{3}\right)_{\Sigma}$ & 1 & -8 & 24 & 1 & 16 & 72 & & -24 & 36 & & & \\
\hline$m^{2}\left(\overline{3}, 2,-\frac{\overline{7}}{6}\right)_{\Sigma}$ & 1 & 12 & -16 & 1 & 36 & 32 & & -24 & 16 & & & \\
\hline$m^{2}\left(\overline{6}, 1,-\frac{1}{3}\right)_{\Sigma}$ & 1 & -8 & -16 & 1 & 16 & 32 & & 16 & 16 & & & \\
\hline$m^{2}\left(8,2,+\frac{1}{2}\right)_{\Sigma}$ & 1 & -8 & 4 & 1 & 16 & 52 & & -4 & -24 & & & \\
\hline$m^{2}\left(1,2,+\frac{1}{2}\right)_{\text {mix }}$ & & & & & & & & & & -3 & $-3 \sqrt{3}$ & $-\sqrt{3}$ \\
\hline$m^{2}\left(3,1,-\frac{1}{3}\right)_{\operatorname{mix}}$ & & & & & & & & & & $2 \sqrt{3}$ & -4 & 2 \\
\hline
\end{tabular}

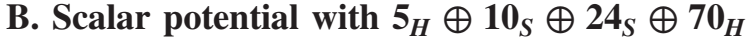

As long as we are interested only in the $v_{\mathrm{GUT}}$-proportional spectrum, the form of the scalar potential remains unaltered upon replacing $\Sigma_{k}^{i j}$ with $\Omega_{k}^{i j}$ in Eqs. (14)-(19). The corresponding scalar spectrum is shown in Tables VIII and X.

There are two major differences from the previous case with $\mathbf{4 5}_{H}$. As before, disabling the mixing terms in the scalar potential introduces the linear dependence among the masses of $\mathbf{7 0}_{H}$,

$$
\begin{aligned}
& m^{2}\left(1,2,+\frac{1}{2}\right)_{\Omega}-\frac{9}{8} m^{2}\left(3,1,-\frac{1}{3}\right)_{\Omega}-\frac{3}{8} m^{2}\left(\overline{3}, 3,+\frac{4}{3}\right)_{\Omega}+\frac{3}{8} m^{2}\left(6,2,-\frac{7}{6}\right)_{\Omega} \\
& \quad+\frac{1}{4} m^{2}\left(8,2,+\frac{1}{2}\right)_{\Omega}-\frac{1}{8} m^{2}\left(1,4,+\frac{1}{2}\right)_{\Omega}=0 \\
& m^{2}\left(3,3,-\frac{1}{3}\right)_{\Omega}+\frac{1}{2} m^{2}\left(\overline{3}, 3,+\frac{4}{3}\right)_{\Omega}-\frac{1}{2} m^{2}\left(6,2,-\frac{7}{6}\right)_{\Omega}+\frac{1}{2} m^{2}\left(15,1,-\frac{1}{3}\right)_{\Omega} \\
& \quad-m^{2}\left(8,2,+\frac{1}{2}\right)_{\Omega}-\frac{1}{2} m^{2}\left(1,4,+\frac{1}{2}\right)_{\Omega}=0
\end{aligned}
$$

but now this dependence is preserved even after the states $\left(1,2,+\frac{1}{2}\right)_{\Omega}$ and $\left(3,1,-\frac{1}{3}\right)_{\Omega}$ effectively decouple through the mixing with $\mathbf{5}_{H}$. As can be seen from Eq. (27) the rest of the states remain linearly dependent, and since some of them are heavy (e.g. the leptoquarks) a certain fine-tuning is needed to make a particular state light as required by unification.

\begin{tabular}{|c|c|c|c|c|c|c|c|c|c|c|c|c|}
\hline & $m_{\Omega}^{2}$ & $\mu_{\Omega}$ & $\mu_{\Omega}^{\prime}$ & $\tilde{\eta}_{1}$ & $\tilde{\eta}_{2}$ & $\tilde{\eta}_{3}$ & $\tilde{\eta}_{4}$ & $\tilde{\eta}_{5}$ & $\tilde{\eta}_{6}$ & $\tilde{\tau}$ & $\tilde{\kappa}_{1}$ & $\tilde{\kappa}_{2}$ \\
\hline$m^{2}\left(1,2,+\frac{1}{2}\right)_{\Omega}$ & 1 & 2 & 14 & 1 & 26 & 62 & 6 & 16 & 6 & & & \\
\hline$m^{2}\left(3,1,-\frac{1}{3}\right)_{\Omega}$ & 1 & $\frac{16}{3}$ & $-\frac{8}{3}$ & 1 & $\frac{88}{3}$ & $\frac{136}{3}$ & $\frac{16}{3}$ & $\frac{28}{3}$ & $-\frac{32}{3}$ & & & \\
\hline$m^{2}\left(3,3,-\frac{1}{3}\right)_{\Omega}$ & 1 & 12 & 4 & 1 & 36 & 52 & & 6 & -24 & & & \\
\hline$m^{2}\left(\overline{3}, 3,+\frac{4}{3}\right)_{\Omega}$ & 1 & -8 & 24 & 1 & 16 & 72 & & -24 & 36 & & & \\
\hline$m^{2}\left(6,2,-\frac{7}{6}\right)_{0}$ & 1 & 12 & -16 & 1 & 36 & 32 & & -24 & 16 & & & \\
\hline$m^{2}\left(15,1,-\frac{1}{3}\right)_{\Omega}$ & 1 & -8 & -16 & 1 & 16 & 32 & & 16 & 16 & & & \\
\hline$m^{2}\left(8,2,+\frac{1}{2}\right)_{\Omega}$ & 1 & -8 & 4 & 1 & 16 & 52 & & -4 & -24 & & & \\
\hline$m^{2}\left(1,4,+\frac{1}{2}\right)_{\Omega}$ & 1 & 12 & 24 & 1 & 36 & 72 & & 36 & 36 & & & \\
\hline $\begin{array}{l}m^{2}\left(1,2,+\frac{1}{2}\right)_{\text {mix }} \\
m^{2}\left(3,1,-\frac{1}{3}\right)_{\text {mix }}\end{array}$ & & & & & & & & & & $\begin{array}{c}-3 \sqrt{2} \\
-4\end{array}$ & $\begin{array}{c}-3 \sqrt{6} \\
\frac{8}{\sqrt{3}}\end{array}$ & $\begin{array}{l}-\sqrt{6} \\
-\frac{4}{\sqrt{3}}\end{array}$ \\
\hline
\end{tabular}

TABLE X. Additional contribution to the scalar spectrum in the vacuum after adding $\mathbf{7 0}_{H}$ to the $\mathbf{5}_{H} \oplus \mathbf{1 0}_{S} \oplus \mathbf{2 4}_{S}$ model. The last two rows represent the mixing between $\mathbf{5}_{H}$ and $\mathbf{7 0}_{H}$. Again, $\mu_{\Omega}, \mu_{\Omega}^{\prime}$ and $\tilde{\tau}$ should be understood as multiplied by $v_{\mathrm{GUT}}$ and $\tilde{\eta}_{1}, \tilde{\eta}_{2}, \tilde{\eta}_{3}, \tilde{\eta}_{4}, \tilde{\eta}_{5}, \tilde{\eta}_{6}, \tilde{\kappa}_{1}$ and $\tilde{\kappa}_{2}$ by $v_{\mathrm{GUT}}^{2}$. 
The second difference comes from the fact that, when considering the full scalar potential for $\mathbf{4 5}_{H}$ and $\mathbf{7 0}_{H}$, they are not of the same form any more due to different symmetry properties of $\Sigma_{k}^{i j}$ and $\Omega_{k}^{i j}$. Namely, since $\phi^{i j}$ is antisymmetric and $\Omega_{k}^{i j}$ is symmetric, all terms contracting $\Omega_{k}^{i j}$ with $\phi_{i j}^{*}$ vanish. Consequently, the Georgi-Jarlskog mechanism cannot be used in this case and we have to rely on either nonrenormalizable Yukawa terms or some other mechanism to explain the pattern of SM fermion masses.

\section{CONCLUSIONS}

Although the SM particle set has been completed with the discovery of the Higgs boson in 2012, it is far from being established as a unique, isolated set [42]. Our search for possible additional particles proceeds with an aim to both explain the neutrino masses and to achieve the unification of gauge couplings. With this in mind, we rely on the BSM states employed in the selected Zee-type BPR neutrino model [26]. This set of states allows us to introduce incomplete $\mathrm{SU}(5)$ representations, that have a potential to improve gauge coupling crossing. Still, this set alone leads to too low unification scale $M_{\mathrm{GUT}}<10^{15} \mathrm{GeV}$, if the Higgs doublet is restricted to belong to $\mathbf{5}_{H}$ irrep.

In contrast, there are immense possibilities to achieve the successful unification if the SM Higgs doublet is embedded into $\mathbf{4 5}_{H}$. Therefore we specify a plausible set of criteria under which our search algorithm shrinks the number of possibilities to seven successful scenarios listed in Table V. In all of them, a light colored scalar $(8,2,1 / 2)$ provided by $\mathbf{4 5}_{H}$ plays a decisive role. If we choose the SM Higgs belonging to $\mathbf{7 0}_{H}$ instead of $\mathbf{4 5}_{H}$, our search algorithm selects four scenarios (A1, A3, A4 and A6) from Table V, and allows for three additional scenarios displayed in Table VII. Notably, in these new scenarios the BPR vectorlike leptons are assigned to complete irrep $\mathbf{5}_{F}$, which do not affect the RGE running. Since in these latter scenarios only the scalar SU(5) irreps are incomplete, an eventual verification of them would be in support of a conjecture [43] that only scalar irreps may be split.

To conclude, in our procedure of renormalizable SU(5) embedding, the colorless BPR particles employed in the neutrino mass model get accompanied by the colored partners to enable a successful unification. We decide to keep sufficiently heavy those among the colored leptoquark scalars which present a threat to proton stability, and the other colored states may play a model-monitoring role both through the LHC phenomenology [34,35] and through tests at Super(and future Hyper)-Kamiokande [37] experiments.

We also point out that in most of the allowed parameter space the color octet scalar $(8,2,1 / 2)$ is the most promising BSM state for the LHC searches, and as such is studied already in [25]. Additional colored states in the specific gauge unification scenarios in Tables V and VII call for a study of characteristic exotic signals at the LHC, which may make some among these specific models falsifiable.

\section{ACKNOWLEDGMENTS}

We would like to thank Borut Bajc, Ilja Doršner, Michal Malinský and Vasja Susič for helpful discussions and correspondence at various stages of development of this project. This work is supported by the Croatian Science Foundation under Project No. 8799 and by the QuantiXLie Center of Excellence KK.01.1.1.01.0004. I. P. also acknowledges partial support by the Croatian Science Foundation Project No. 4418.

\section{APPENDIX A: ALGORITHM FOR OPTIMAL UNIFICATION SEARCH}

When studying GUT models with several new BSM states, one needs a well-defined procedure for identifying the viable unification scenarios. To this end, it is of some advantage to "linearize" the B-test (12),

$$
\frac{B_{23}}{B_{12}}=0.718 \equiv b,
$$

by first separating the fixed contribution of SM states from a contribution of the variable mass BSM states,

$$
B_{i j}=B_{i j}^{\mathrm{SM}}+B_{i j}^{\mathrm{BSM}} .
$$

In the next step we write the gauge coupling crossing condition in the form

$$
B_{23}^{\mathrm{BSM}}-b B_{12}^{\mathrm{BSM}}=b B_{12}^{\mathrm{SM}}-B_{23}^{\mathrm{SM}}=1.384 \equiv c .
$$

Finally, we separate the $\beta$-function coefficients $\Delta b_{i}^{(k)}$ from the threshold weight factors $r_{k}$ (11) of each of the $N$ BSM states and write the crossing test as

$$
\sum_{k=1}^{N} c_{k} r_{k}=c,
$$

where $c_{k}=\left(\Delta b_{2}^{(k)}-\Delta b_{3}^{(k)}\right)-b\left(\Delta b_{1}^{(k)}-\Delta b_{2}^{(k)}\right)$. For example, the $E_{L, R}, h^{+}$and $\Delta$ states responsible for the neutrino masses in the BPR model, if they are at electroweak scale $\left(r_{k} \approx 1\right)$, contribute to this sum with $\sum c_{k} r_{k} \approx 3$, and significantly overshoot the required value (A3). Thus, we need to add extra states with total negative contribution $\sum c_{k} r_{k} \approx-1.6$. By choosing some particular set of states we solve for $r_{k}$.

In principle, there is an experimental uncertainty of constant $c$ [related to the uncertainty of $b$ in (A1)], but we do not need to discuss it because most of the time we will be able to require the exact gauge crossing, regardless of possible small variations in the value of $c$. 
Along the same lines, the expression for the GUT scale (13) can be recast in a condition on $B_{12}^{\mathrm{BSM}}$,

$$
B_{12}^{\mathrm{BSM}}=\frac{184.87}{\ln M_{\mathrm{GUT}} / m_{Z}}-B_{12}^{\mathrm{SM}} \approx-1.43 \equiv s,
$$

where to get the numerical value we use the low experimental bound on $M_{\mathrm{GUT}}=5 \times 10^{15} \mathrm{GeV}$. This can again be written in a simple form, linear in variables $r_{k}$,

$$
\sum_{k=1}^{N} s_{k} r_{k}=s,
$$

with $s_{k}=\Delta b_{1}^{(k)}-\Delta b_{2}^{(k)}$.

Obviously, if we have just one new variable BSM state $(\mathrm{N}=1)$ at our disposal, we can just solve the crossing condition (A4), obeying any existing experimental lower bounds

$$
m_{k} \geq m_{k, \text { min }} \rightarrow r_{k} \leq r_{k, \text { max }},
$$

and check that the GUT scale is high enough. For two states, $N=2, M_{\mathrm{GUT}}$ can also be chosen at will, and we can either require it equal to the experimental lowest bound, or look for the range of possible $M_{\mathrm{GUT}}$ for which the solution of (A4) and (A6) exists. Regardless of this choice, for more than two particles, $N \geq 3$, the problem becomes underdetermined and we need another criteria. To obtain definite scenarios we choose to maximize the norm of the vector,

$$
\mathbf{r}=\left(r_{1}, r_{2}, \ldots, r_{N}\right)
$$

which means that we choose scenarios with roughly minimal masses of new particles, or, in other words, we choose scenarios which have maximal discovery potential.

Following further this principle of maximal discovery potential, one could also try to minimize at the same time the distance of $M_{\mathrm{GUT}}$ to the existing experimental lower bound. We have tried this, but a necessary choice of relative weight of two optimization objectives brings a complication which we deem unnecessary at this point. Thus, we performed, for each choice for a set of BSM states, a onedimensional scan with ever increasing fixed $M_{\mathrm{GUT}}$, to find the range of $M_{\mathrm{GUT}}$ for which the unification scenario works. The problem can be organized as a standard linear algebra matrix equation,

$\mathbf{A r} \equiv\left(\begin{array}{cccc}c_{1} & c_{2} & \cdots & c_{N} \\ s_{1} & s_{2} & \cdots & s_{N}\end{array}\right)\left(\begin{array}{c}r_{1} \\ r_{2} \\ \cdots \\ r_{N}\end{array}\right)=\left(\begin{array}{c}c \\ s\end{array}\right) \equiv \mathbf{a}$,

and if we are temporarily not concerned with bounds on $r_{k}$, it can be solved using the Lagrange multiplier method to obtain a solution with extremal $\|\mathbf{r}\|$, which is $\mathbf{r}=\mathbf{A}^{\top}\left(\mathbf{A A}^{\top}\right)^{-1} \mathbf{a}$. One can also choose to make a variable change $r_{k} \rightarrow x_{k} \equiv r_{k, \max }-r_{k}$ and minimize $\|\mathbf{x}\|$ instead of maximizing $\|\mathbf{r}\|$. To take the bounds on $r_{k}$ properly into account, a more sophisticated optimization algorithm is needed and we used the sequential least squares programming algorithm SLSQP [44,45].

\section{APPENDIX B: DETAILS OF SU(5) REPRESENTATIONS}

In this Appendix we present the structure and normalizations of used SU(5) representations.

\section{Adjoint representation}

$\chi_{j}^{i}=\left(\begin{array}{ccccc}-\frac{2}{\sqrt{30}} \sigma+\frac{1}{\sqrt{2}} O_{1}+\frac{1}{\sqrt{6}} O_{2} & O_{R \bar{G}} & O_{R \bar{B}} & X_{R} & Y_{R} \\ O_{G \bar{R}} & -\frac{2}{\sqrt{30}} \sigma-\frac{1}{\sqrt{2}} O_{1}+\frac{1}{\sqrt{6}} O_{2} & O_{G \bar{B}} & X_{G} & Y_{G} \\ O_{B \bar{R}} & O_{B \bar{G}} & -\frac{2}{\sqrt{30}} \sigma-\frac{2}{\sqrt{6}} O_{2} & X_{B} & Y_{B} \\ \bar{X}_{R} & \bar{X}_{G} & \bar{X}_{B} & \frac{3}{\sqrt{30}} \sigma+\frac{1}{\sqrt{2}} \Delta_{0} & \Delta_{+} \\ \bar{Y}_{R} & \bar{Y}_{G} & \bar{Y}_{B} & \Delta_{-} & \frac{3}{\sqrt{30}} \sigma-\frac{1}{\sqrt{2}} \Delta_{0}\end{array}\right)$,

is the adjoint 24-dimensional real traceless representation

$$
\sum_{i=1}^{5} \chi_{i}^{i}=0
$$


which decomposes under the SM group as

$$
\begin{aligned}
\mathbf{2 4}= & \overbrace{(1,1,0)}^{\sigma} \oplus \overbrace{(1,3,0)}^{\Delta} \oplus \overbrace{(8,1,0)}^{O} \oplus \overbrace{\left(3,2,-\frac{5}{6}\right)}^{X, Y} \\
& \oplus \overbrace{\left(\overline{3}, 2,+\frac{5}{6}\right)}^{\bar{X}, \bar{Y}} .
\end{aligned}
$$

The symbols $\sigma, \Delta, O, X, Y$ (and their complex conjugates $\bar{X}$ and $\bar{Y}$ ) denote the SM singlet field, the weak triplet, the color octet and the lower and upper components of $\mathrm{SU}(2)_{L}$ doublet $\left(3,2,-\frac{5}{6}\right)$, respectively. Note that the singlet $\sigma$, the electrically neutral triplet component $\Delta_{0}$ and colorless octet components $O_{1}$ and $O_{2}$ are real fields so that their mass terms come with a prefactor $\frac{1}{2}$.

\section{Fundamental representation}

$H^{i}$ and $H_{i}^{*}$ are the five-dimensional fundamental and antifundamental complex representations with SM decomposition

$$
\mathbf{5}=\overbrace{\left(1,2,+\frac{1}{2}\right)}^{H} \oplus \overbrace{\left(3,1,-\frac{1}{3}\right)}^{S_{1}}
$$

whose fields have the same quantum numbers as

$$
\overline{\mathbf{5}}_{F}=\left(\begin{array}{c}
d_{\alpha}^{c} \\
\epsilon_{a b} L^{b}
\end{array}\right)=\left(\begin{array}{c}
d_{\bar{R}}^{c} \\
d_{\bar{G}}^{c} \\
d_{\bar{B}}^{c} \\
e \\
-\nu
\end{array}\right)
$$

and where the weak doublet can play the role of Standard Model Higgs and potentially mix with its counterpart from $\Sigma_{k}^{i j}$ or $\Omega_{k}^{i j}$.

\section{Two-index antisymmetric representation}

$\phi^{i j}$ and $\phi_{i j}^{*}$ are ten-dimensional complex antisymmetric representations

$$
\phi^{i j}=-\phi^{j i},
$$

with the SM decomposition

$$
\mathbf{1 0}=\overbrace{(1,1,+1)}^{h^{+}} \oplus\left(\overline{3}, 1,-\frac{2}{3}\right) \oplus\left(3,2,+\frac{1}{6}\right)
$$

whose fields have the same quantum numbers as

$$
\mathbf{1 0}_{F}=\frac{1}{\sqrt{2}}\left(\begin{array}{cc}
\epsilon^{\alpha \beta \gamma} u_{\gamma}^{c} & Q^{\alpha b} \\
-\left(Q^{\beta a}\right)^{T} & \epsilon^{a b} e^{c}
\end{array}\right)=\frac{1}{\sqrt{2}}\left(\begin{array}{ccccc}
0 & u_{\bar{B}}^{c} & -u_{\bar{G}}^{c} & u_{R} & d_{R} \\
-u_{\bar{B}}^{c} & 0 & u_{\bar{R}}^{c} & u_{G} & d_{G} \\
u_{\bar{G}}^{c} & -u_{\bar{R}}^{c} & 0 & u_{B} & d_{B} \\
-u_{R} & -u_{G} & -u_{B} & 0 & e^{c} \\
-d_{R} & -d_{G} & -d_{B} & -e^{c} & 0
\end{array}\right)
$$

The normalization factor $\frac{1}{\sqrt{2}}$ is there only for convenience to avoid the double counting of fields in the mass term.

\section{45-dimensional representation}

$\Sigma_{k}^{i j}$ and $\Sigma_{i j}^{* k}$ are 45 -dimensional complex representations satisfying the antisymmetry and tracelessness conditions

$$
\begin{gathered}
\Sigma_{k}^{i j}=-\Sigma_{k}^{j i}, \\
\sum_{i=1}^{5} \Sigma_{i}^{i j}=0, \quad j=1, \ldots, 5 .
\end{gathered}
$$

Under the SM it is decomposed as

$$
\mathbf{4 5}=\overbrace{\left(1,2,+\frac{1}{2}\right)}^{\Sigma_{a}} \oplus \overbrace{\left(3,1,-\frac{1}{3}\right)}^{\Sigma_{b}} \oplus \overbrace{\left(3,3,-\frac{1}{3}\right)}^{\Sigma_{c}} \oplus \overbrace{\left(\overline{3}, 1,+\frac{4}{3}\right)}^{\Sigma_{c}} \oplus \overbrace{\left(\overline{3}, 2,-\frac{7}{6}\right)}^{\Sigma_{d}} \oplus \overbrace{\left(\overline{6}, 1,-\frac{1}{3}\right)}^{\Sigma_{e}} \oplus \overbrace{\left(8,2,+\frac{1}{2}\right)}^{\Sigma_{f}},
$$


where its nonzero components are

$$
\begin{aligned}
& \Sigma_{4}^{15}=-\Sigma_{4}^{51} \rightarrow \frac{\Sigma_{c}^{-R}}{\sqrt{2}}, \quad \Sigma_{4}^{25}=-\Sigma_{4}^{52} \rightarrow \frac{\Sigma_{c}^{-G}}{\sqrt{2}}, \quad \Sigma_{4}^{35}=-\Sigma_{4}^{53} \rightarrow \frac{\Sigma_{c}^{-B}}{\sqrt{2}}, \\
& \Sigma_{5}^{14}=-\Sigma_{5}^{41} \rightarrow \frac{\Sigma_{c}^{+R}}{\sqrt{2}}, \quad \Sigma_{5}^{24}=-\Sigma_{5}^{42} \rightarrow \frac{\Sigma_{c}^{+G}}{\sqrt{2}}, \quad \Sigma_{5}^{34}=-\Sigma_{5}^{43} \rightarrow \frac{\Sigma_{c}^{+B}}{\sqrt{2}}, \\
& \Sigma_{1}^{45}=-\Sigma_{1}^{54} \rightarrow \frac{\Sigma_{d}^{\bar{R}}}{\sqrt{2}}, \quad \Sigma_{2}^{45}=-\Sigma_{2}^{54} \rightarrow \frac{\Sigma_{d}^{\bar{G}}}{\sqrt{2}}, \quad \Sigma_{3}^{45}=-\Sigma_{3}^{54} \rightarrow \frac{\Sigma_{d}^{\bar{B}}}{\sqrt{2}}, \\
& \Sigma_{4}^{23}=-\Sigma_{4}^{32} \rightarrow \frac{\Sigma_{e}^{-\bar{R}}}{\sqrt{2}}, \quad \Sigma_{4}^{13}=-\Sigma_{4}^{31} \rightarrow \frac{\Sigma_{e}^{-\bar{G}}}{\sqrt{2}}, \quad \Sigma_{4}^{12}=-\Sigma_{4}^{21} \rightarrow \frac{\Sigma_{e}^{-\bar{B}}}{\sqrt{2}}, \\
& \Sigma_{5}^{23}=-\Sigma_{5}^{32} \rightarrow \frac{\Sigma_{e}^{+\bar{R}}}{\sqrt{2}}, \quad \Sigma_{5}^{13}=-\Sigma_{5}^{31} \rightarrow \frac{\Sigma_{e}^{+\bar{G}}}{\sqrt{2}}, \quad \Sigma_{5}^{12}=-\Sigma_{5}^{21} \rightarrow \frac{\Sigma_{e}^{+\bar{B}}}{\sqrt{2}}, \\
& \Sigma_{1}^{23}=-\Sigma_{1}^{32} \rightarrow \frac{\Sigma_{f}^{\bar{R}} \bar{R}}{\sqrt{2}}, \quad \Sigma_{2}^{13}=-\Sigma_{2}^{31} \rightarrow \frac{\Sigma_{f}^{\bar{G} \bar{G}}}{\sqrt{2}}, \quad \Sigma_{3}^{12}=-\Sigma_{3}^{21} \rightarrow \frac{\Sigma_{f}^{\bar{B} \bar{B}}}{\sqrt{2}}, \\
& \Sigma_{2}^{34}=-\Sigma_{2}^{43} \rightarrow \frac{\Sigma_{g}^{+B \bar{G}}}{\sqrt{2}}, \quad \Sigma_{3}^{14}=-\Sigma_{3}^{41} \rightarrow \frac{\Sigma_{g}^{+R \bar{B}}}{\sqrt{2}}, \quad \Sigma_{1}^{24}=-\Sigma_{1}^{42} \rightarrow \frac{\Sigma_{g}^{+G \bar{R}}}{\sqrt{2}}, \\
& \Sigma_{3}^{24}=-\Sigma_{3}^{42} \rightarrow \frac{\Sigma_{g}^{+G \bar{B}}}{\sqrt{2}}, \quad \Sigma_{1}^{34}=-\Sigma_{1}^{43} \rightarrow \frac{\Sigma_{g}^{+B \bar{R}}}{\sqrt{2}}, \quad \Sigma_{2}^{14}=-\Sigma_{2}^{41} \rightarrow \frac{\Sigma_{g}^{+R \bar{G}}}{\sqrt{2}}, \\
& \Sigma_{2}^{35}=-\Sigma_{2}^{53} \rightarrow \frac{\Sigma_{g}^{-B \bar{G}}}{\sqrt{2}}, \quad \Sigma_{3}^{15}=-\Sigma_{3}^{51} \rightarrow \frac{\Sigma_{g}^{-R \bar{B}}}{\sqrt{2}}, \quad \Sigma_{1}^{25}=-\Sigma_{1}^{52} \rightarrow \frac{\Sigma_{g}^{-G \bar{R}}}{\sqrt{2}}, \\
& \Sigma_{3}^{25}=-\Sigma_{3}^{52} \rightarrow \frac{\Sigma_{g}^{-G \bar{B}}}{\sqrt{2}}, \quad \Sigma_{1}^{35}=-\Sigma_{1}^{53} \rightarrow \frac{\Sigma_{g}^{-B \bar{R}}}{\sqrt{2}}, \quad \Sigma_{2}^{15}=-\Sigma_{2}^{51} \rightarrow \frac{\Sigma_{g}^{-R \bar{G}}}{\sqrt{2}}, \\
& \Sigma_{2}^{12}=-\Sigma_{2}^{21} \rightarrow \frac{\Sigma_{b}^{R}}{2 \sqrt{2}}+\frac{\Sigma_{f}^{R}}{2}, \quad \Sigma_{3}^{23}=-\Sigma_{3}^{32} \rightarrow \frac{\Sigma_{b}^{G}}{2 \sqrt{2}}-\frac{\Sigma_{f}^{G}}{2}, \\
& \Sigma_{1}^{13}=-\Sigma_{1}^{31} \rightarrow-\frac{\Sigma_{b}^{B}}{2 \sqrt{2}}+\frac{\Sigma_{f}^{B}}{2}, \quad \Sigma_{3}^{13}=-\Sigma_{3}^{31} \rightarrow \frac{\Sigma_{b}^{R}}{2 \sqrt{2}}-\frac{\Sigma_{f}^{R}}{2}, \\
& \Sigma_{1}^{12}=-\Sigma_{1}^{21} \rightarrow-\frac{\Sigma_{b}^{G}}{2 \sqrt{2}}-\frac{\Sigma_{f}^{G}}{2}, \quad \Sigma_{2}^{23}=-\Sigma_{2}^{32} \rightarrow-\frac{\Sigma_{b}^{B}}{2 \sqrt{2}}-\frac{\Sigma_{f}^{B}}{2}, \\
& \Sigma_{4}^{14}=-\Sigma_{4}^{41} \rightarrow-\frac{\Sigma_{b}^{R}}{2 \sqrt{2}}+\frac{\Sigma_{c}^{0 R}}{2}, \quad \Sigma_{4}^{24}=-\Sigma_{4}^{42} \rightarrow-\frac{\Sigma_{b}^{G}}{2 \sqrt{2}}+\frac{\Sigma_{c}^{0 G}}{2}, \\
& \Sigma_{4}^{34}=-\Sigma_{4}^{43} \rightarrow-\frac{\Sigma_{b}^{B}}{2 \sqrt{2}}-\frac{\Sigma_{c}^{0 B}}{2}, \quad \Sigma_{5}^{15}=-\Sigma_{5}^{51} \rightarrow-\frac{\Sigma_{b}^{R}}{2 \sqrt{2}}-\frac{\Sigma_{c}^{0 R}}{2}, \\
& \Sigma_{5}^{25}=-\Sigma_{5}^{52} \rightarrow-\frac{\Sigma_{b}^{G}}{2 \sqrt{2}}-\frac{\Sigma_{c}^{0 G}}{2}, \quad \Sigma_{5}^{35}=-\Sigma_{5}^{53} \rightarrow-\frac{\Sigma_{b}^{B}}{2 \sqrt{2}}+\frac{\Sigma_{c}^{0 B}}{2}, \\
& \Sigma_{1}^{14}=-\Sigma_{1}^{41} \rightarrow \frac{\Sigma_{a}^{+}}{2 \sqrt{6}}+\frac{\Sigma_{g}^{+S_{1}}}{2}+\frac{\Sigma_{g}^{+S_{2}}}{2 \sqrt{3}}, \quad \Sigma_{1}^{15}=-\Sigma_{1}^{51} \rightarrow-\frac{\Sigma_{a}^{-}}{2 \sqrt{6}}+\frac{\Sigma_{g}^{-S_{1}}}{2}+\frac{\Sigma_{g}^{-S_{2}}}{2 \sqrt{3}}, \\
& \Sigma_{2}^{24}=-\Sigma_{2}^{42} \rightarrow \frac{\Sigma_{a}^{+}}{2 \sqrt{6}}-\frac{\Sigma_{g}^{+S_{1}}}{2}+\frac{\Sigma_{g}^{+S_{2}}}{2 \sqrt{3}}, \quad \Sigma_{2}^{25}=-\Sigma_{2}^{52} \rightarrow-\frac{\Sigma_{a}^{-}}{2 \sqrt{6}}-\frac{\Sigma_{g}^{-S_{1}}}{2}+\frac{\Sigma_{g}^{-S_{2}}}{2 \sqrt{3}}, \\
& \Sigma_{3}^{34}=-\Sigma_{3}^{43} \rightarrow \frac{\Sigma_{a}^{+}}{2 \sqrt{6}}-\frac{\Sigma_{g}^{+S_{2}}}{\sqrt{3}}, \quad \Sigma_{3}^{35}=-\Sigma_{3}^{53} \rightarrow-\frac{\Sigma_{a}^{-}}{2 \sqrt{6}}-\frac{\Sigma_{g}^{-S_{2}}}{\sqrt{3}}, \\
& \Sigma_{5}^{45}=-\Sigma_{5}^{54} \rightarrow \frac{1}{2} \sqrt{\frac{3}{2}} \Sigma_{a}^{+}, \quad \Sigma_{4}^{45}=-\Sigma_{4}^{54} \rightarrow \frac{1}{2} \sqrt{\frac{3}{2}} \Sigma_{a}^{-},
\end{aligned}
$$


with the superscript used to distinguish the individual field components in $\Sigma_{a, \ldots, h}$ by indicating the sign of their $S U(2)_{L}$ and their $S U(3)_{c}$ quantum numbers [where $R, G$ and $B$ stand for $\left(\frac{1}{2}, \frac{1}{2 \sqrt{3}}\right),\left(-\frac{1}{2}, \frac{1}{2 \sqrt{3}}\right)$ and $\left(0,-\frac{1}{\sqrt{3}}\right)$ pairs] under the diagonal generators of the corresponding subgroup. In this notation it is the field $\Sigma_{a}^{-}$[a neutral component of $\left.\left(1,2,+\frac{1}{2}\right)_{\Sigma}\right]$ which develops a nonzero $\operatorname{VEV}\left\langle\Sigma_{a}^{-}\right\rangle \equiv v_{45}$.

\section{70-dimensional representation}

$\Omega_{k}^{i j}$ and $\Omega_{i j}^{* k}$ are 70 -dimensional complex representations satisfying the symmetry and tracelessness conditions

$$
\begin{gathered}
\Omega_{k}^{i j}=\Omega_{k}^{j i}, \\
\sum_{i=1}^{5} \Omega_{i}^{i j}=0, \quad j=1, \ldots, 5 .
\end{gathered}
$$

Under SM it gets decomposed as

$$
\begin{aligned}
& \mathbf{7 0}=\overbrace{\left(1,2,+\frac{1}{2}\right)}^{\Omega_{a}} \oplus \overbrace{\left(3,1,-\frac{1}{3}\right)}^{\Omega_{b}} \oplus \overbrace{\left(3,3,-\frac{1}{3}\right)}^{\Omega_{c}} \oplus \overbrace{\left(\overline{3}, 3,+\frac{4}{3}\right)}^{\Omega_{d}} \oplus \overbrace{\left(6,2,-\frac{7}{6}\right)}^{\Omega_{e}} \\
& \oplus \underbrace{\left(15,1,-\frac{1}{3}\right)}_{\Omega_{f}} \oplus \underbrace{\left(8,2,+\frac{1}{2}\right)}_{\Omega_{g}} \oplus \underbrace{\left(1,4,+\frac{1}{2}\right)}_{\Omega_{h}}
\end{aligned}
$$

where its nonzero components are

$$
\begin{aligned}
& \Omega_{1}^{11} \rightarrow \frac{\Omega_{b}^{R}}{\sqrt{6}}-\frac{\Omega_{f}^{R_{2}}}{\sqrt{2}}, \quad \Omega_{1}^{22} \rightarrow \Omega_{f}^{G G \bar{R}}, \quad \Omega_{1}^{33} \rightarrow \Omega_{f}^{B B \bar{R}}, \quad \Omega_{1}^{44} \rightarrow \Omega_{d}^{+\bar{R}}, \quad \Omega_{1}^{55} \rightarrow \Omega_{d}^{-\bar{R}}, \\
& \Omega_{2}^{11} \rightarrow \Omega_{f}^{R R \bar{G}}, \quad \Omega_{2}^{22} \rightarrow \frac{\Omega_{b}^{G}}{\sqrt{6}}-\frac{\Omega_{f}^{G_{2}}}{\sqrt{2}}, \quad \Omega_{2}^{33} \rightarrow \Omega_{f}^{B B \bar{G}}, \quad \Omega_{2}^{44} \rightarrow \Omega_{d}^{+\bar{G}}, \quad \Omega_{2}^{55} \rightarrow \Omega_{d}^{-\bar{G}}, \\
& \Omega_{3}^{11} \rightarrow \Omega_{f}^{R R \bar{B}}, \quad \Omega_{3}^{22} \rightarrow \Omega_{f}^{G G \bar{B}}, \quad \Omega_{3}^{33} \rightarrow \frac{\Omega_{b}^{B}}{\sqrt{6}}-\frac{\Omega_{f}^{B_{2}}}{\sqrt{2}}, \quad \Omega_{3}^{44} \rightarrow \Omega_{d}^{+\bar{B}}, \quad \Omega_{3}^{55} \rightarrow \Omega_{d}^{-\bar{B}}, \\
& \Omega_{4}^{11} \rightarrow \Omega_{e}^{-R R}, \quad \Omega_{4}^{22} \rightarrow \Omega_{e}^{-G G}, \quad \Omega_{4}^{33} \rightarrow \Omega_{e}^{-B B}, \quad \Omega_{4}^{44} \rightarrow-\frac{\Omega_{a}^{+}}{\sqrt{3}}+\frac{\Omega_{h}^{+}}{\sqrt{3}}, \quad \Omega_{4}^{55} \rightarrow \Omega_{h}^{--}, \\
& \Omega_{5}^{11} \rightarrow \Omega_{e}^{+R R}, \quad \Omega_{5}^{22} \rightarrow \Omega_{e}^{+G G}, \quad \Omega_{5}^{33} \rightarrow \Omega_{e}^{+B B}, \quad \Omega_{5}^{44} \rightarrow \Omega_{h}^{++}, \quad \Omega_{5}^{55} \rightarrow \frac{\Omega_{a}^{-}}{\sqrt{3}}-\frac{\Omega_{h}^{-}}{\sqrt{3}}, \\
& \Omega_{4}^{15}=\Omega_{4}^{51} \rightarrow \frac{\Omega_{c}^{-R}}{\sqrt{2}}, \quad \Omega_{4}^{25}=\Omega_{4}^{52} \rightarrow \frac{\Omega_{c}^{-G}}{\sqrt{2}}, \quad \Omega_{4}^{35}=\Omega_{4}^{53} \rightarrow \frac{\Omega_{c}^{-B}}{\sqrt{2}}, \\
& \Omega_{5}^{14}=\Omega_{5}^{41} \rightarrow \frac{\Omega_{c}^{+R}}{\sqrt{2}}, \quad \Omega_{5}^{24}=\Omega_{5}^{42} \rightarrow \frac{\Omega_{c}^{+G}}{\sqrt{2}}, \quad \Omega_{5}^{34}=\Omega_{5}^{43} \rightarrow \frac{\Omega_{c}^{+B}}{\sqrt{2}}, \\
& \Omega_{1}^{45}=\Omega_{1}^{54} \rightarrow \frac{\Omega_{d}^{0 \bar{R}}}{\sqrt{2}}, \quad \Omega_{2}^{45}=\Omega_{2}^{54} \rightarrow \frac{\Omega_{d}^{0 \bar{G}}}{\sqrt{2}}, \quad \Omega_{3}^{45}=\Omega_{3}^{54} \rightarrow \frac{\Omega_{d}^{0 \bar{B}}}{\sqrt{2}}, \\
& \Omega_{4}^{23}=\Omega_{4}^{32} \rightarrow \frac{\Omega_{e}^{-\bar{R}}}{\sqrt{2}}, \quad \Omega_{4}^{13}=\Omega_{4}^{31} \rightarrow \frac{\Omega_{e}^{-\bar{G}}}{\sqrt{2}}, \quad \Omega_{4}^{12}=\Omega_{4}^{21} \rightarrow \frac{\Omega_{e}^{-\bar{B}}}{\sqrt{2}}, \\
& \Omega_{5}^{23}=\Omega_{5}^{32} \rightarrow \frac{\Omega_{e}^{+\bar{R}}}{\sqrt{2}}, \quad \Omega_{5}^{13}=\Omega_{5}^{31} \rightarrow \frac{\Omega_{e}^{+\bar{G}}}{\sqrt{2}}, \quad \Omega_{5}^{12}=\Omega_{5}^{21} \rightarrow \frac{\Omega_{e}^{+\bar{B}}}{\sqrt{2}}, \\
& \Omega_{1}^{23}=\Omega_{1}^{32} \rightarrow \frac{\Omega_{f}^{\bar{R} \bar{R}}}{\sqrt{2}}, \quad \Omega_{2}^{13}=\Omega_{2}^{31} \rightarrow \frac{\Omega_{f}^{\bar{G} \bar{G}}}{\sqrt{2}}, \quad \Omega_{3}^{12}=\Omega_{3}^{21} \rightarrow \frac{\Omega_{f}^{\bar{B} \bar{B}}}{\sqrt{2}},
\end{aligned}
$$




$$
\begin{aligned}
& \Omega_{2}^{34}=\Omega_{2}^{43} \rightarrow \frac{\Omega_{g}^{+B \bar{G}}}{\sqrt{2}}, \quad \Omega_{3}^{14}=\Omega_{3}^{41} \rightarrow \frac{\Omega_{g}^{+R \bar{B}}}{\sqrt{2}}, \quad \Omega_{1}^{24}=\Omega_{1}^{42} \rightarrow \frac{\Omega_{g}^{+G \bar{R}}}{\sqrt{2}}, \\
& \Omega_{3}^{24}=\Omega_{3}^{42} \rightarrow \frac{\Omega_{g}^{+G \bar{B}}}{\sqrt{2}}, \quad \Omega_{1}^{34}=\Omega_{1}^{43} \rightarrow \frac{\Omega_{g}^{+B \bar{R}}}{\sqrt{2}}, \quad \Omega_{2}^{14}=\Omega_{2}^{41} \rightarrow \frac{\Omega_{g}^{+R \bar{G}}}{\sqrt{2}}, \\
& \Omega_{2}^{35}=\Omega_{2}^{53} \rightarrow \frac{\Omega_{g}^{-B \bar{G}}}{\sqrt{2}}, \quad \Omega_{3}^{15}=\Omega_{3}^{51} \rightarrow \frac{\Omega_{g}^{-R \bar{B}}}{\sqrt{2}}, \quad \Omega_{1}^{25}=\Omega_{1}^{52} \rightarrow \frac{\Omega_{g}^{-G \bar{R}}}{\sqrt{2}}, \\
& \Omega_{3}^{25}=\Omega_{3}^{52} \rightarrow \frac{\Omega_{g}^{-G \bar{B}}}{\sqrt{2}}, \quad \Omega_{1}^{35}=\Omega_{1}^{53} \rightarrow \frac{\Omega_{g}^{-B \bar{R}}}{\sqrt{2}}, \quad \Omega_{2}^{15}=\Omega_{2}^{51} \rightarrow \frac{\Omega_{g}^{-R \bar{G}}}{\sqrt{2}}, \\
& \Omega_{2}^{12}=\Omega_{2}^{21} \rightarrow \frac{\Omega_{b}^{R}}{2 \sqrt{6}}+\frac{\Omega_{f}^{R_{1}}}{2}+\frac{\Omega_{f}^{R_{2}}}{2 \sqrt{2}}, \quad \Omega_{3}^{23}=\Omega_{3}^{32} \rightarrow \frac{\Omega_{b}^{G}}{2 \sqrt{6}}-\frac{\Omega_{f}^{G_{1}}}{2}+\frac{\Omega_{f}^{G_{2}}}{2 \sqrt{2}}, \\
& \Omega_{1}^{13}=\Omega_{1}^{31} \rightarrow \frac{\Omega_{b}^{B}}{2 \sqrt{6}}+\frac{\Omega_{f}^{B_{1}}}{2}+\frac{\Omega_{f}^{B_{2}}}{2 \sqrt{2}}, \quad \Omega_{3}^{13}=\Omega_{3}^{31} \rightarrow \frac{\Omega_{b}^{R}}{2 \sqrt{6}}-\frac{\Omega_{f}^{R_{1}}}{2}+\frac{\Omega_{f}^{R_{2}}}{2 \sqrt{2}}, \\
& \Omega_{1}^{12}=\Omega_{1}^{21} \rightarrow \frac{\Omega_{b}^{G}}{2 \sqrt{6}}+\frac{\Omega_{f}^{G_{1}}}{2}+\frac{\Omega_{f}^{G_{2}}}{2 \sqrt{2}}, \quad \Omega_{2}^{23}=\Omega_{2}^{32} \rightarrow \frac{\Omega_{b}^{B}}{2 \sqrt{6}}-\frac{\Omega_{f}^{B_{1}}}{2}+\frac{\Omega_{f}^{B_{2}}}{2 \sqrt{2}}, \\
& \Omega_{4}^{14}=\Omega_{4}^{41} \rightarrow-\frac{\Omega_{b}^{R}}{\sqrt{6}}+\frac{\Omega_{c}^{0 R}}{2}, \quad \Omega_{4}^{24}=\Omega_{4}^{42} \rightarrow-\frac{\Omega_{b}^{G}}{\sqrt{6}}+\frac{\Omega_{c}^{0 G}}{2}, \\
& \Omega_{4}^{34}=\Omega_{4}^{43} \rightarrow-\frac{\Omega_{b}^{B}}{\sqrt{6}}-\frac{\Omega_{c}^{0 B}}{2}, \quad \Omega_{5}^{15}=\Omega_{5}^{51} \rightarrow-\frac{\Omega_{b}^{R}}{\sqrt{6}}-\frac{\Omega_{c}^{0 R}}{2}, \\
& \Omega_{5}^{25}=\Omega_{5}^{52} \rightarrow-\frac{\Omega_{b}^{G}}{\sqrt{6}}-\frac{\Omega_{c}^{0 G}}{2}, \quad \Omega_{5}^{35}=\Omega_{5}^{53} \rightarrow-\frac{\Omega_{b}^{B}}{\sqrt{6}}+\frac{\Omega_{c}^{0 B}}{2}, \\
& \Omega_{1}^{14}=\Omega_{1}^{41} \rightarrow \frac{\Omega_{a}^{+}}{2 \sqrt{3}}+\frac{\Omega_{g}^{+S_{1}}}{2}+\frac{\Omega_{g}^{+S_{2}}}{2 \sqrt{3}}, \quad \Omega_{1}^{15}=\Omega_{1}^{51} \rightarrow-\frac{\Omega_{a}^{-}}{2 \sqrt{3}}+\frac{\Omega_{g}^{-S_{1}}}{2}+\frac{\Omega_{g}^{-S_{2}}}{2 \sqrt{3}}, \\
& \Omega_{2}^{24}=\Omega_{2}^{42} \rightarrow \frac{\Omega_{a}^{+}}{2 \sqrt{3}}-\frac{\Omega_{g}^{+S_{1}}}{2}+\frac{\Omega_{g}^{+S_{2}}}{2 \sqrt{3}}, \quad \Omega_{2}^{25}=\Omega_{2}^{52} \rightarrow-\frac{\Omega_{a}^{-}}{2 \sqrt{3}}-\frac{\Omega_{g}^{-S_{1}}}{2}+\frac{\Omega_{g}^{-S_{2}}}{2 \sqrt{3}}, \\
& \Omega_{3}^{34}=\Omega_{3}^{43} \rightarrow \frac{\Omega_{a}^{+}}{2 \sqrt{3}}-\frac{\Omega_{g}^{+S_{2}}}{\sqrt{3}}, \quad \Omega_{3}^{35}=\Omega_{3}^{53} \rightarrow-\frac{\Omega_{a}^{-}}{2 \sqrt{3}}-\frac{\Omega_{g}^{-S_{2}}}{\sqrt{3}}, \\
& \Omega_{5}^{45}=\Omega_{5}^{54} \rightarrow-\frac{\Omega_{a}^{+}}{2 \sqrt{3}}-\frac{\Omega_{h}^{+}}{\sqrt{3}}, \quad \Omega_{4}^{45}=\Omega_{4}^{54} \rightarrow \frac{\Omega_{a}^{-}}{2 \sqrt{3}}+\frac{\Omega_{h}^{-}}{\sqrt{3}},
\end{aligned}
$$

and the same notation for individual field components was used as for $\Sigma_{k}^{i j}$ [with the exception of the weak quartet $\left(1,4,+\frac{1}{2}\right)$ fields $\Omega_{h}^{--}, \Omega_{h}^{-}, \Omega_{h}^{+}$and $\Omega_{h}^{++}$denoted with respect to their increasing $S U(2)_{L}$ quantum number $-\frac{3}{2},-\frac{1}{2},+\frac{1}{2}$ and $\left.+\frac{3}{2}\right]$. In the chosen notation the neutral components of $\left(1,2,+\frac{1}{2}\right)_{\Omega}$ and $\left(1,4,+\frac{1}{2}\right)_{\Omega}$ can develop a weak-scale $\operatorname{VEV}\left\langle\Omega_{a}^{-}\right\rangle$and $\left\langle\Omega_{h}^{-}\right\rangle$, respectively.

[1] P. Minkowski, $\mu \rightarrow e \gamma$ at a rate of one out of $10^{9}$ muon decays?, Phys. Lett. B 67, 421 (1977).

[2] T. Yanagida, Horizontal symmetry and masses of neutrinos, Conference Proceedings C7902131, 95 (1979).

[3] M. Gell-Mann, P. Ramond, and R. Slansky, Complex spinors and unified theories, Conf. Proc. C 790927, 315 (1979).
[4] S. L. Glashow, The future of elementary particle physics, NATO Sci. Ser. B 61, 687 (1980).

[5] R. N. Mohapatra and G. Senjanović, Neutrino Mass and Spontaneous Parity Violation, Phys. Rev. Lett. 44, 912 (1980).

[6] W. Konetschny and W. Kummer, Nonconservation of total lepton number with scalar bosons, Phys. Lett. B 70, 433 (1977). 
[7] M. Magg and C. Wetterich, Neutrino mass problem and gauge hierarchy, Phys. Lett. B 94, 61 (1980).

[8] J. Schechter and J. W. F. Valle, Neutrino masses in $\mathrm{SU}(2) \times$ U(1) theories, Phys. Rev. D 22, 2227 (1980).

[9] T.P. Cheng and L.-F. Li, Neutrino masses, mixings and oscillations in $\mathrm{SU}(2) \times \mathrm{U}(1)$ models of electroweak interactions, Phys. Rev. D 22, 2860 (1980).

[10] G. Lazarides, Q. Shafi, and C. Wetterich, Proton lifetime and fermion masses in an SO(10) model, Nucl. Phys. B181, 287 (1981).

[11] R. N. Mohapatra and G. Senjanović, Neutrino masses and mixings in gauge models with spontaneous parity violation, Phys. Rev. D 23, 165 (1981).

[12] R. Foot, H. Lew, X. G. He, and G. C. Joshi, Seesaw neutrino masses induced by a triplet of leptons, Z. Phys. C 44, 441 (1989).

[13] H. Georgi and S. L. Glashow, Unity of All Elementary Particle Forces, Phys. Rev. Lett. 32, 438 (1974).

[14] U. Amaldi, W. de Boer, and H. Furstenau, Comparison of grand unified theories with electroweak and strong coupling constants measured at LEP, Phys. Lett. B 260, 447 (1991).

[15] L.-F. $\mathrm{Li}$ and F. Wu, Coupling constant unification in extensions of standard model, Int. J. Mod. Phys. A 19, 3217 (2004).

[16] N. V. Krasnikov, (Non)supersymmetric SU(5) grand unified models with light colored octets and electroweak triplets, Phys. Lett. B 306, 283 (1993).

[17] S. Willenbrock, Triplicated trinification, Phys. Lett. B 561, 130 (2003).

[18] B. Bajc and G. Senjanović, Seesaw at LHC, J. High Energy Phys. 08 (2007) 014.

[19] B. Bajc, M. Nemevšek, and G. Senjanović, Probing seesaw at LHC, Phys. Rev. D 76, 055011 (2007).

[20] I. Doršner and P. Fileviez Perez, Upper bound on the mass of the type III seesaw triplet in an SU(5) model, J. High Energy Phys. 06 (2007) 029.

[21] I. Doršner, P. Fileviez Perez, and R. Gonzalez Felipe, Phenomenological and cosmological aspects of a minimal GUT scenario, Nucl. Phys. B747, 312 (2006).

[22] I. Doršner and P. Fileviez Perez, Unification without supersymmetry: Neutrino mass, proton decay and light leptoquarks, Nucl. Phys. B723, 53 (2005).

[23] I. Dorsner and I. Mocioiu, Predictions from type II see-saw mechanism in SU(5), Nucl. Phys. B796, 123 (2008).

[24] A. Zee, A theory of lepton number violation, neutrino Majorana mass, and oscillation, Phys. Lett. B 93, 389 (1980); Erratum, Phys. Lett. B 95, 461 (1980).

[25] P. Fileviez Perez and C. Murgui, Renormalizable SU(5) unification, Phys. Rev. D 94, 075014 (2016).

[26] V. Brdar, I. Picek, and B. Radovčić, Radiative neutrino mass with scotogenic scalar triplet, Phys. Lett. B 728, 198 (2014).

[27] P. Čuljak, K. Kumerički, and I. Picek, Scotogenic R $\nu$ MDM at three-loop level, Phys. Lett. B 744, 237 (2015).
[28] A. Ahriche, K. L. McDonald, S. Nasri, and T. Toma, A model of neutrino mass and dark matter with an accidental symmetry, Phys. Lett. B 746, 430 (2015).

[29] D. Aristizabal Sierra, C. Simoes, and D. Wegman, Closing in on minimal dark matter and radiative neutrino masses, J. High Energy Phys. 06 (2016) 108.

[30] O. Antipin, P. Čuljak, K. Kumerički, and I. Picek, Extended Higgs sectors in radiative neutrino models, Phys. Lett. B 768, 330 (2017).

[31] M. Heikinheimo, K. Kannike, F. Lyonnet, M. Raidal, K. Tuominen, and H. Veermäe, Vacuum stability and perturbativity of SU(3) scalars, J. High Energy Phys. 10 (2017) 014.

[32] H. Georgi and C. Jarlskog, A new lepton-Quark mass relation in a unified theory, Phys. Lett. B 86, 297 (1979).

[33] J. Kopp, M. Lindner, V. Niro, and T. E. J. Underwood, On the consistency of perturbativity and gauge coupling unification, Phys. Rev. D 81, 025008 (2010).

[34] A. Giveon, L. J. Hall, and U. Sarid, SU(5) unification revisited, Phys. Lett. B 271, 138 (1991).

[35] I. Doršner, S. Fajfer, D. A. Faroughy, and N. Košnik, The role of the $S_{3}$ GUT leptoquark in flavor universality and collider searches, J. High Energy Phys. 10 (2017) 188.

[36] C. Patrignani et al. (Particle Data Group Collaboration), Review of particle physics, Chin. Phys. C 40, 100001 (2016).

[37] K. Abe et al. (Super-Kamiokande Collaboration), Search for proton decay via $p \rightarrow e^{+} \pi^{0}$ and $p \rightarrow \mu^{+} \pi^{0}$ in 0.31 megaton years exposure of the Super-Kamiokande water Cherenkov detector, Phys. Rev. D 95, 012004 (2017).

[38] A. M. Sirunyan et al. (CMS Collaboration), Search for dijet resonances in proton-proton collisions at $\sqrt{s}=13 \mathrm{TeV}$ and constraints on dark matter and other models, Phys. Lett. B 769, 520 (2017); Corrigendum, Phys. Lett. B 772, 882 (2017)

[39] T. Han, I. Lewis, and Z. Liu, Colored resonant signals at the LHC: Largest rate and simplest topology, J. High Energy Phys. 12 (2010) 085.

[40] R. Sekhar Chivukula, E. H. Simmons, and N. Vignaroli, Distinguishing dijet resonances at the LHC, Phys. Rev. D 91, 055019 (2015).

[41] A. Hayreter and G. Valencia, LHC constraints on color octet scalars, Phys. Rev. D 96, 035004 (2017).

[42] J. D. Wells, Z. Zhang, and Y. Zhao, Establishing the isolated standard model, Phys. Rev. D 96, 015005 (2017).

[43] P. Cox, A. Kusenko, O. Sumensari, and T. T. Yanagida, SU(5) unification with TeV-scale leptoquarks, J. High Energy Phys. 03 (2017) 035.

[44] D. Kraft, A software package for sequential quadratic programming, Technical Report No. DFVLR-FB 88-28, DLR German Aerospace Center, 1988.

[45] In the interest of open and reproducible research, the computer code used in the production of numerical results for this paper is made available at https://github.com/ openhep/kmp17. 